\title{
Advection\#dominated Accretion Model of Sagittarius A*: Evidence for a Black Hole at the Galactic Center
}

\section{Citation}

Narayan, Ramesh, Rohan Mahadevan, Jonathan E. Grindlay, Robert G. Popham, and Charles Gammie. 1998. "Advection\#dominated Accretion Model of Sagittarius A*: Evidence for a Black Hole at the Galactic Center." The Astrophysical Journal 492 (2): 554-68. https:// doi.org/10.1086/305070.

\section{Permanent link}

http://nrs.harvard.edu/urn-3:HUL.InstRepos:41384913

\section{Terms of Use}

This article was downloaded from Harvard University's DASH repository, and is made available under the terms and conditions applicable to Other Posted Material, as set forth at http:// nrs.harvard.edu/urn-3:HUL.InstRepos:dash.current.terms-of-use\#LAA

\section{Share Your Story}

The Harvard community has made this article openly available.

Please share how this access benefits you. Submit a story.

\section{Accessibility}




\title{
Advection-Dominated Accretion Model of Sagittarius A*: Evidence for a Black Hole at the Galactic Center
}

\author{
Ramesh Narayan, Rohan Mahadevan, Jonathan E. Grindlay, Robert G. Popham, \\ Charles Gammie \\ Harvard-Smithsonian Center for Astrophysics, 60 Garden St., Cambridge, MA 02138
}

\begin{abstract}
Sgr A* at the Galactic Center is a puzzling source. It has a mass $M=(2.5 \pm$ $0.4) \times 10^{6} M_{\odot}$ which makes it an excellent black hole candidate. Observations of stellar winds and other gas flows in its vicinity suggest a mass accretion rate $\dot{M} \gtrsim$ few $\times 10^{-6} M_{\odot} \mathrm{yr}^{-1}$. However, such an accretion rate would imply a luminosity $>10^{40} \mathrm{erg} \mathrm{s}^{-1}$ if the radiative efficiency is the usual $10 \%$, whereas observations indicate a bolometric luminosity $<10^{37} \mathrm{erg} \mathrm{s}^{-1}$. The spectrum of Sgr $A^{*}$ is unusual, with emission extending over many decades of wavelength. We present a model of Sgr $\mathrm{A}^{*}$ which is based on a two-temperature optically-thin advection-dominated accretion flow. The model is consistent with the estimated $M$ and $\dot{M}$, and fits the observed fluxes in the $\mathrm{cm} / \mathrm{mm}$ and X-ray bands as well as upper limits in the sub-mm and infrared bands; the fit is less good in the radio below $86 \mathrm{GHz}$ and in $\gamma$-rays above $100 \mathrm{MeV}$. The very low luminosity of Sgr $A^{*}$ is explained naturally in the model by means of advection. Most of the viscously dissipated energy is advected into the central mass by the accreting gas, and therefore the radiative efficiency is extremely low, $\sim 5 \times 10^{-6}$. A critical element of the model is the presence of an event horizon at the center which swallows the advected energy. The success of the model could thus be viewed as confirmation that $\operatorname{Sgr} \mathrm{A}^{*}$ is a black hole.
\end{abstract}

Subject headings: accretion, accretion disks — black holes — galaxies: nuclei - Galaxy: center — radiation mechanisms: bremsstrahlung, inverse Compton, synchrotron - radio sources: Sgr A*

\section{Introduction}

The enigmatic radio source, Sagittarius A* (Sgr A*), has for many years been a puzzle (Genzel \& Townes 1987; Genzel, Hollenbach \& Townes 1994; Mezger, Duschl \& Zylka 
1996). The source is located at the dynamical center of the Galaxy and is presumed to be associated with a supermassive black hole. However, observations provide conflicting indications both for and against the black hole hypothesis and there is currently no model that explains all the observations. The paradoxical clues may be summarized briefly as follows:

1. Dynamical measurements indicate a dark mass of $\sim(2.5 \pm 0.4) \times 10^{6} M_{\odot}$ within the central 0.1 pc of the Galactic Center (Haller et al. 1996; Eckart \& Genzel 1997). This is believed to be the mass $M$ of the putative supermassive black hole in Sgr A*.

2. Observations of stellar winds and gas flows near Sgr $\mathrm{A}^{*}$, coupled with the above estimate of the black hole mass, suggest that Sgr $\mathrm{A}^{*}$ must accrete gas from its surrounding at a mass accretion rate $\dot{M} \gtrsim$ few $\times 10^{-6} M_{\odot} \mathrm{yr}^{-1}$ (Genzel et al. 1994).

3. This $\dot{M}$ implies an accretion luminosity $L \sim 0.1 \dot{M} c^{2}>10^{40} \mathrm{erg} \mathrm{s}^{-1}$, assuming a nominal radiative efficiency of $10 \%$. However, $\operatorname{Sgr} A^{*}$ is an unusually dim source with a total luminosity from radio to $\gamma$-rays under $10^{37} \mathrm{erg} \mathrm{s}^{-1}$. The extremely low luminosity has been used to argue against Sgr A* being an accreting black hole (Goldwurm et al. 1994).

4. For the above $M$ and $\dot{M}$ the peak emission from a standard thin accretion disk will be in the near infrared (cf. Frank, King \& Raine 1992). In fact, this is a generic prediction of any model that involves an optically thick flow radiating as a blackbody. However, Menten et al. (1997) have obtained a strong upper limit on the 2.2 micron flux of Sgr $\mathrm{A}^{*}$ that effectively rules out such models.

5. Sgr $\mathrm{A}^{*}$ is brightest in the radio/mm band (see $\S 2$ and Fig. 1), it is weakly detected in X-rays (Predehl \& Trumper 1994), and it may have been detected between $100 \mathrm{MeV}$ and $2 \mathrm{GeV}$ (Merck et al. 1996). These observations, combined with the Menten et al. infrared upper limit, imply a spectral distribution completely unlike a blackbody or even a sum of power-law components. The observations suggest that $\operatorname{Sgr} \mathrm{A}^{*}$ is optically thin, and that many different radiation processes may be in operation.

Over the years a number of models have been proposed for Sgr A*. Some of these have been phenomenological approaches which aim to explain the radio and infrared spectrum without including any detailed dynamics (e.g. Falcke 1996, Duschl \& Lesch 1994, Beckert \& Duschl 1997). These models generally require mass accretion rates lower than those indicated by the observations. Other models (Melia 1992, 1994; Mastichiadis \& Ozernoy 1994) have attempted to incorporate dynamics, but with simplifying assumptions such as ignoring the angular momentum of the accreting gas. One model of Sgr $\mathrm{A}^{*}$ that has attempted a self-consistent treatment of both viscous hydrodynamics and radiation processes is the advection-dominated accretion flow (ADAF) model of Narayan, Yi \& Mahadevan 
(1995). An early and qualitative discussion of this model was presented by Rees (1982). The present paper is based on the ADAF model.

An advection-dominated accretion flow is one in which most of the energy released by viscous dissipation is stored in the gas and advected to the center, and only a small fraction of the energy is radiated (Narayan \& Yi 1994, 1995a, 1995b; Chen et al. 1995; see Narayan 1997 for a recent review). Most current work on ADAFs has been concerned with a branch of low $\dot{M}$ solutions (Ichimaru 1977; Rees et al. 1982; Narayan \& Yi 1995b; Abramowicz et al. 1995) which is present for mass accretion rates below a few percent of the Eddington rate (Narayan 1997; Esin, McClintock \& Narayan 1997). These low- $\dot{M}$ solutions make use of the standard $\alpha$ viscosity and assume a two-temperature plasma with an equipartition magnetic field. The two-temperature paradigm was introduced to astrophysics by Shapiro, Lightman \& Eardley (1976; see also Phinney 1981 and Rees et al. 1982) and is based on the following two assumptions: (1) It is assumed that the bulk of the viscous energy is deposited in the ions, with only a small fraction of the energy going directly into the electrons. (2) Energy transfer from the ions to the electrons is assumed to occur via Coulomb collisions, with no significant nonthermal coupling being present (see Phinney 1981; Begelman \& Chiueh 1988). In the low- $\dot{M}$ two-temperature ADAF model the ions achieve a nearly virial temperature, $T_{i} \sim 10^{12} \mathrm{~K} / r$, where $r$ is the radius in Schwarzschild units, while the electron temperature saturates at around $10^{9}-10^{10} \mathrm{~K}$ for $r \lesssim 100$ (Narayan \& Yi 1995b). The high ion temperature causes the gas to take up a quasi-spherical shape (Narayan \& Yi 1995a), the gas is optically thin, and the flow does not suffer from any serious thermal or viscous instabilities (Narayan \& Yi 1995b; Abramowicz et al. 1995; Kato, Abramowicz \& Chen 1996).

The low- $\dot{M}$ branch of ADAFs has several appealing features for explaining the observations of Sgr $\mathrm{A}^{*}$ and other low-luminosity accretion systems. First, since most of the energy is advected with the gas and lost into the black hole, the model naturally explains the low luminosity of Sgr $A^{*}$. Second, being optically thin, the spectrum is quite different from blackbody. Indeed, the high electron and ion temperatures allow a variety of radiation processes to operate: synchrotron, bremsstrahlung and inverse Compton from the electrons (Narayan \& Yi 1995b; Mahadevan 1997), and $\gamma$-ray emission from the ions via pion production (Mahadevan, Narayan \& Krolik 1997). Therefore, the gas is likely to radiate over a wide range of wavelengths.

The Narayan et al. (1995) ADAF model of Sgr A* gave a reasonable fit to the observations available at that time and provided an explanation for the low luminosity of the source. However, the model required a black hole mass of $7 \times 10^{5} M_{\odot}$ which differs from the most recent dynamical mass estimate of $2.5 \times 10^{6} M_{\odot}$ (Eckart \& Genzel 1997). This 
problem prompted us to take another look at the model.

In addition, there have been important developments on the observational and theoretical front which further motivate the present study. The recent Menten et al. (1997) limit on the infrared flux of Sgr A* is well below previous "detections" (Rosa et al. 1991; Eckart et al. 1992) which were used by Narayan et al. (1995) to fit the ADAF model. Similarly, ASCA observations by Koyama et al. (1996) have shown that the X-ray emission from the Galactic Center region is dominated by an X-ray burster which lies within 1.3 arcminutes of Sgr $A^{*}$. This implies that most previous measurements of the X-ray flux of Sgr A* (Pavlinskii, Grebenev, \& Sunyaev 1992, 1994; Skinner et al. 1987) are suspect since they were obtained with inadequate spatial resolution. Only the ROSAT PSPC detection by Predehl \& Trumper (1994) survives as a reliable X-ray detection, although the corresponding luminosity of the source in the ROSAT band is uncertain due to uncertainties in the absorbing hydrogen column (cf. §2).

The modeling techniques have also advanced significantly during the intervening two years. The Narayan et al. (1995) model was a local one where the dynamics were calculated using a local self-similar solution (Narayan \& Yi 1994) and the radiation was computed with a local approximation for Compton scattering (Dermer, Liang \& Canfield 1991; Narayan \& Yi 1995b). Consistent global dynamical solutions have since been calculated, initially with a pseudo-Newtonian potential (Narayan, Kato \& Honma 1997; Chen, Abramowicz \& Lasota 1997) and more recently for the full relativistic Kerr problem (Abramowicz et al. 1996; Peitz \& Appl 1997; Gammie \& Popham 1997). A global scheme for Comptonization has also been developed (Narayan, Barret \& McClintock 1997), based on techniques due to Poutanen \& Svensson (1996). Finally, Nakamura et al. (1997) have shown that in addition to energy advection by ions, which had been considered in previous work, advection by electrons can be important in some circumstances. This effect is now included in the calculations (Appendix A). The present models are thus superior to those used by Narayan et al. (1995).

In this paper we present improved ADAF models of Sgr A*. We make use of the spectral data described in $\S 2$ and employ the modeling techniques outlined in $\S 3$. We present detailed results in $\S 4$ and conclude with a discussion in $\S 5$.

\section{Spectral Data}

The radio to near infrared (NIR) spectrum of Sgr $\mathrm{A}^{*}$ has been of constant interest since the discovery of the source by Balick \& Brown (1974), and observations have been carried out from $400 \mathrm{MHz}$ (Davies et al. 1976) to $\sim 10^{14} \mathrm{~Hz}$ (Menten et al. 1997). Table 
1 is a compilation of the radio to NIR observations. The data are also plotted in Fig. 1. The two general features in the table and figures are 1) we have given only the maximum and minimum fluxes for frequencies at which variability has been observed, and 2) open circles correspond to low resolution measurements, which we treat as upper limits, while filled circles correspond to the highest resolution measurements in each band (subject to the additional constraint that we require the resolution to be $<1$ arcmin). The latter measurements, which are identified with a $\star$ in Table 1 , are most likely to probe the actual accretion flow in Sgr A*, while the former could be contaminated by emission from other components in the source. This is discussed in detail below.

In determining the spectrum of the ADAF from Sgr $A^{*}$, special attention has to be paid to the angular resolution of the observations, which ideally must be comparable to the size of the ADAF. The angular size of the ADAF in Sgr A* depends on the wavelength band under consideration. The low frequency $(<1 \mathrm{GHz})$ radio emission is from radii on the order of $\sim 10^{3} R_{s}-10^{4} R_{s}$ (where $R_{s}$ is the Schwarzschild radius), which corresponds to an angular size at the Galactic Center of $\lesssim 0.06^{\prime \prime}(\sim 500 \mathrm{AU})$, where we have taken $M=2.5 \times 10^{6} M_{\odot}$. With increasing frequency, the size becomes smaller, and the sub-mm and infrared emission are from no more than a few tens of $R_{s}$. The soft X-rays are again from a large volume (up to about $10^{4} R_{s}$ ), but harder X-rays are from progressively smaller radii. Gamma-ray emission from pion decay is again limited to a few tens of $R_{s}$.

The radio spectrum of Sgr $A^{*}$ appears to consist of two components, with a break at around the $\nu \sim 86 \mathrm{GHz}$ point in Fig. 1. The spectral dependence is $L_{\nu} \sim \nu^{0.2}$ for $\nu<40 \mathrm{GHz}$, and steepens to $L_{\nu} \sim \nu^{0.8}$ above $40 \mathrm{GHz}$. The steep increase has been noted by Zylka et al. (1992, 1995), Serabyn et al. $(1992,1997)$ and Rogers et al. (1995), but the origin of the two components is unclear. Perhaps coincidentally, for frequencies $\lesssim 50 \mathrm{GHz}$, scattering by turbulent density fluctuations in the ISM leads to source broadening. The apparent size of the source, which is proportional to the square of the observed wavelength (e.g. Davies et al. 1976; Backer 1982), is larger than the intrinsic size at these frequencies. The low frequency data could thus in principle be contaminated by structures in the source (e.g. jets) which are larger than the relevant radiating region of the ADAF but smaller than the scatter-broadened size.

The ADAF model we present in the following sections fits the data above $86 \mathrm{GHz}$ well but underpredicts the flux at lower frequencies. Motivated by this, and in view of the scattering argument mentioned above, we plot the low frequency data as open circles in Fig. 1. We treat these data as upper bounds rather than as firm detections of the central accretion flow in Sgr A*. Thus, even though the Marcaide et al. (1992) and Alberdi et al. (1993) measurements at $22 \mathrm{GHz}$ had exceptionally good resolution, we still plot these points 
as open circles because they are affected by scattering. At $22 \mathrm{GHz}$, the scattered source size is $\sim 15 \mathrm{AU}$, whereas the relevant region of the ADAF which produces the radiation has a size of $\sim 1 \mathrm{AU}$. Any contamination to the radio flux must be present on a scale $\lesssim 15 \mathrm{AU}$, which is rather a stringent requirement. We must therefore keep open the possibility that the low frequency radiation (i.e. below the apparent break at $\sim 86 \mathrm{GHz}$ ) also arises from the accretion flow. The models presented in this paper are then unable to account for the observed level of this low frequency emission.

For frequencies $\gtrsim 50 \mathrm{GHz}$, the scattering size falls below the intrinsic size. High resolution observations can therefore determine directly the emission from the central engine. However, only a few high resolution ( $\lesssim 1$ mas) observations have been made at these wavelengths using VLBI or the VLBA. These radio observations are represented as filled circles in Fig. 1. We take the point at $86 \mathrm{GHz}$ to be the best determined radio flux of $\mathrm{Sgr} \mathrm{A}^{*}$ for the purposes of modeling the source. Apart from the VLBI/VLBA points, all other radio points $\gtrsim 86 \mathrm{GHz}$ have been represented by open circles since these observations were done with poor resolution $\left(\gtrsim 4^{\prime \prime}\right)$.

The FIR to NIR observations are all upper limits, and the low angular resolution points are represented by open circles. The upper limits by Gezari et al. (1994) and Zylka et al. (1992) are the best resolution observations in the FIR band, and are therefore shown as filled circles. Stolovy et al. (1996) detected a source in the mid-infrared with an extinctioncorrected flux of $100 \pm 40$ mJy. However, their resolution was $0.5^{\prime \prime}$ and their flux may possibly be contaminated by the compact cluster of sources observed by Eckart et al. (1995). We plot their data point as an upper limit with a filled circle. The recent upper limit of Menten et al. (1997) in the NIR at $2.2 \mu \mathrm{m}$ is a very high resolution measurement since the authors were able to determine accurately the relative position of Sgr $A^{*}$ in their speckle images by comparing with a radio map of nearby stellar $\mathrm{SiO}$ masers. The effective resolution of the speckle image is $0.15^{\prime \prime}$, and therefore we plot this limit again as a filled circle.

$\mathrm{X}$-ray and hard X-ray as well as gamma-ray observations of Sgr A* have long been limited by the relatively poor angular resolution available at these energies and the very crowded field of the Galactic Center region. Even at hard X-ray energies (e.g. 20-100 keV) where the density of sources is relatively low, the Sgr A* source region contains more sources $(\sim 10)$ in the central $5^{\circ}$ of the Galaxy than any other region (Goldwurm et al. 1994). At soft X-ray (e.g. 0.5-4 keV) and low-medium X-ray energies (e.g. 2-10 keV), this same region is even more crowded, with complex diffuse emission and at least 10 sources within the central $1^{\circ}$ of Sgr A* (cf. Watson et al 1991 and Koyama et al 1996). Thus the flux and spectral distribution of the Galactic Center in the $\sim 1-100 \mathrm{keV}$ band, where the ADAF bremsstrahlung component peaks (cf. Fig. 1), is particularly critical and angular resolution 
is paramount. At the soft X-ray end of this band, the relatively uncertain absorbing column density, $N_{H}$, towards the Sgr A* source is especially important since the value chosen (which can in principle be determined by future high resolution observations with AXAF) greatly affects the derived source flux and luminosity.

We have included in the $\mathrm{X}$-ray/hard X-ray region of the spectrum plotted in Figure 1 only 4 points or upper limits which all are the highest angular resolution available in their respective energy bands. These data are listed in Table 2. The one detection of a point-like source with position fully consistent with Sgr A* is the ROSAT detection (PSPC) with $\sim 20^{\prime \prime}$ resolution in the $0.8-2.5 \mathrm{keV}$ band reported by Predehl and Trumper (1994). This detection is plotted for an assumed $N_{H}=6 \times 10^{22} \mathrm{~cm}^{-2}$, which is the usual best estimate (cf. Watson et al 1981) for the interstellar column density and which corresponds to the usually quoted visual extinction of $\mathrm{A}_{V} \sim 25-30 \mathrm{mag}$. For this $N_{H}$, the total integrated luminosity in the $0.8-2.5 \mathrm{keV}$ band is $L_{X}=1.5 \times 10^{34} \mathrm{erg} \mathrm{s}^{-1}$. T The $N_{H}$ used here differs from the much higher value $\left(1.5 \times 10^{23} \mathrm{~cm}^{-2}\right)$ assumed by Predehl and Trumper. These authors chose a higher $N_{H}$ in order to make the soft $\mathrm{X}$-ray luminosity more compatible with the variable compact source within $\sim 1^{\prime}$ of Sgr A* reported by Skinner et al (1987) and Pavlinskii, Grebenev and Sunyaev (1992) from 2-20 keV coded aperture imaging observations with modest (few arcmin) resolution. However, recent ASCA observations of the Galactic center in the 2-10 keV band, with $\sim 1^{\prime}$ angular resolution but much higher spectral resolution, have been reported by Koyama et al. (1996) and Maeda et al (1996). They show the Sgr $\mathrm{A}^{*}$ region to be complex, with diffuse emission over a $\sim 2 \times 3^{\prime}$ region and a point source at $1.3^{\prime}$ from $\mathrm{Sgr} \mathrm{A}^{*}$ which is an $\mathrm{X}$-ray burster and therefore a neutron star in a $(8.4 \mathrm{~h})$ binary system. The burster has very likely dominated the coded aperture imaging flux measurements. Koyama et al. therefore quote an upper limit of $10^{36} \mathrm{erg} / \mathrm{s}$ as the $2-10 \mathrm{keV}$ luminosity for the entire Sgr $\mathrm{A}^{*}$ complex, with a possible actual value of $\sim 10^{35} \mathrm{erg} / \mathrm{s}$ for the point source alone. We have plotted this ASCA upper limit in Fig. 1.

The ROSAT PSPC detection, although only with angular resolution of $\sim 20^{\prime \prime}$, is a likely detection of Sgr $\mathrm{A}^{*}$ with minimal contamination from the surrounding diffuse source since it appears in the ROSAT image to be consistent with a point source. However, higher spatial resolution (e.g. AXAF) X-ray imaging, and/or evidence for time variability, are needed to confirm this identification. In this sense, even the ROSAT detection might be regarded as an upper limit. Nevertheless, this data point is plotted as a solid symbol as it corresponds to the highest resolution X-ray observation. The luminosity plotted in Fig. 1 and listed in Table 2 corresponds to the likely $N_{H}$ value of $6 \times 10^{22} \mathrm{~cm}^{-2}$ and was obtained by considering

\footnotetext{
${ }^{1}$ The $L_{X}$ and $\nu L_{\nu}$ calculations were done for the ROSAT data using the PIMMS program, supplied by the HEASARC at GSFC.
} 
two extreme values of a power law spectral index: photon index 1.0 and 2.0. These indices bracket the corresponding index $(\sim 1.4)$ for a bremsstrahlung spectrum as predicted by our model. The detected flux in the soft $(0.8-2.5 \mathrm{keV})$ band is, however, still highly sensitive to the assumed $N_{H}$ (as also pointed out by Predehl and Trumper): for $N_{H}=8 \times 10^{22}$ the flux increases by a factor of 2.8 over that shown; whereas for $N_{H}=5 \times 10^{22}$, it decreases by a factor of 0.6. Thus the fits to ADAF models described in the next section, which are otherwise greatly constrained by this ROSAT detection, should be regarded as uncertain in normalization by a factor perhaps as large as 2 if uncertainties in the interstellar $N_{H}$ are allowed for completely and perhaps as large as 3 if internal self-absorption in the vicinity of the AGN is allowed for (as suggested by Predehl and Trumper). The box around the ROSAT point in Fig. 1 includes both the uncertainty due to spectral index as well as an additional factor of 2 uncertainty in normalizaton. The uncertain value of $N_{H}$ can, in principle, be directly measured or at least greatly constrained by observations of Sgr A* with the ACIS instrument on AXAF. This could observe the interstellar absorption edge of Oxygen (at $0.8 \mathrm{keV}$ ) at the high angular resolution $\left(1^{\prime \prime}\right)$ needed to isolate completely the surrounding diffuse emission.

At still higher energies, 20-100 keV, we plot the two upper limits derived from the deep SIGMA observations of the Galactic Center complex reported by Goldwurm et al. (1994). These observations are not able to resolve the burster, and are likely affected by it (or, rather, constrain the hard X-ray flux from the burster). More sensitive observations in the hard X-ray band at energies above 60-100 keV, where the hard X-ray spectral component of neutron stars in bursters usually is not detectable (e.g. Barret \& Grindlay 1995) could provide a more stringent test of the ADAF spectrum as well as the predicted spectral turnover.

The $\gamma$-ray spectrum shown in Fig. 1 corresponds to emission from the EGRET source 2EG 1746-2852 which is coincident with the Galactic Center (Merck et al. 1996). The source is described as point-like to within the resolution of the instrument $\left(\sim 1^{\circ}\right)$, and is a significant excess $(\sim 10 \sigma)$ above the local diffuse emission. The source has a very hard spectrum with a photon index of 1.7 which differs significantly from other unidentified Galactic EGRET sources. The source might therefore represent emission from Sgr A*, but it could equally well be unresolved emission from a giant molecular cloud in the vicinity of the Galactic Center. If the source is Sgr A*, the flux and spectrum may be explained as $\gamma$-ray emission via pion production and decay in the ADAF (Mahadevan et al. 1997). If the source is instead associated with a dense cloud of molecular hydrogen situated at the Galactic Center, it will again show a pion decay spectrum but with a spectral index (at energies above $100 \mathrm{MeV}$ ) given by the local cosmic ray proton spectrum (i.e. a power law with energy index $\sim-1.6$ ). Due to the low resolution of EGRET ( $1^{\circ}$ beam) and uncertainty 
in the nature of the source, we plot the $\gamma$-ray data in Fig. 1 as open circles. The EGRET observations (as reported by Mayer-Hasselwander, 4th Compton Symp.) indicate possible variability, which would of course suggest that the source is indeed Sgr A*, but the same EGRET data also indicate that the source may be slightly resolved, which would argue that the radiation is significantly contaminated by surrounding diffuse emission. Future observations with higher sensitivity and better resolution are needed to resolve this issue.

\section{Modeling Techniques}

We consider a black hole of mass $M$ accreting through an ADAF at a rate $\dot{m} \dot{M}_{\text {Edd }}$, where $\dot{M}_{\mathrm{Edd}}=10 L_{\mathrm{Edd}} / c^{2}=1.39 \times 10^{18}\left(M / M_{\odot}\right) \mathrm{g} \mathrm{s}^{-1}=2.21 \times 10^{-8}\left(M / M_{\odot}\right) M_{\odot} \mathrm{yr}^{-1}$. An ADAF is in some sense dynamically intermediate between a thin disk and a spherical accretion flow: it is hot and quasi-spherical $(H / R \sim 1$, Narayan \& Yi 1995a), with rapid radial inflow, yet centrifugal support and viscous angular momentum transport still play a significant role. In describing the ADAF we shall generally refer to the scaled radius $r \equiv R / R_{s}$, where $R_{s}=2 G M / c^{2}$ is the Schwarzschild radius. We assume that the ADAF extends from the black hole horizon, $r=1$, to an outer radius $r_{\text {out }}$. We take the mean angular momentum vector of the ADAF to be inclined at an angle $i$ to the line of sight.

To find the dynamical structure of the ADAF, we use the fully relativistic, selfconsistent, steady-state global solutions in the Kerr metric developed by Popham \& Gammie (1997). Their model uses a nearly standard viscosity prescription which is parameterized by the efficiency of angular momentum transport, $\alpha$ (assumed constant, independent of radius). The viscosity prescription has been modified, however, so as to preserve causality and to include relativistic effects. We also use a quasi-spherical prescription for the vertical structure based on Abramowicz, Lanza, and Percival (1997). Comparison of relativistic and nonrelativistic global models, and the self-similar solution of Narayan \& Yi (1994) shows that they are rather similar outside about $r=5$. Inside this radius the density in our solution is very close to self-similar, but the temperature is slightly lower.

The dynamical model is uniquely specified by four structure parameters: $\alpha$, the viscos-

ity parameter; $\gamma$, the adiabatic index of the fluid, which is assumed to be a mixture of gas and magnetic fields; $f$, the advection parameter, which gives the ratio of advected energy to the viscous heat input; and $a$, the rotation parameter of the black hole. Of these four parameters, $\gamma$ is determined in terms of the magnetic field equipartition parameter $\beta$ (defined below) according to (Esin 1997): $\gamma=(8-3 \beta) /(6-3 \beta)$. The viscosity parameter $\alpha$ is again determined in terms of $\beta$ following Hawley, Gammie \& Balbus (1996): $\alpha=0.6(1-\beta)$. The parameter $f$ is determined self-consistently by iterating between the dynamical solution 
and the radiation solution and seeking consistency between the two (see Esin et al. 1997 for details). The models considered here are extremely advection-dominated, so that $f$ is very close to unity in all cases. (For instance, for the baseline model shown in Fig. 1, we obtain $f=0.9994$.) Finally, we consider only the case $\mathrm{a}=0$, i.e. a Schwarzschild black hole.

The thermodynamic state of the flow is described by the ion temperature $T_{i}$, the electron temperature $T_{e}$, and the magnetic pressure $p_{\text {mag }} \equiv(1-\beta) p_{\text {tot }}$. The total pressure is fixed by the dynamical model. We assume that $\beta$ is a constant, independent of radius, and so $\alpha$ and $\gamma$ are also independent of radius. Thus, all the structural parameters are constant in our model (cf. §3). Given $\beta$ we know the run of gas pressure with radius. Since the electrons can cool efficiently and coupling between the ions and electrons is assumed to be weak (provided solely by Coulomb collisions), we have $T_{i} \gg T_{e}$. The run of gas pressure then gives $T_{i}$ to a good approximation.

The heart of the problem is now calculating the electron temperature, which determines the spectral properties of the flow. A detailed description of our procedure is given in Esin et al. (1997). Basically, the electron temperature is determined by solving an energy balance equation:

$$
Q^{\mathrm{e}, \mathrm{adv}}=Q^{\mathrm{ie}}+\delta Q^{\mathrm{vis}}-Q^{\mathrm{rad}}
$$

Here $Q^{\text {eadv }}$ is the rate at which energy in the electrons is advected inward by the flow. This term is discussed in greater detail in Appendix A. Our earlier work (with the exception of Esin et al. 1997) ignored the advective term, but recently Nakamura et al. (1997) (see also Mahadevan \& Quataert 1997) have shown that it can be important, particularly at low mass accretion rates. At low $\dot{m}$ the electrons are nearly adiabatic and the compressive heating as the gas flows in becomes large. The other terms in equation (1) are as follows: $Q^{\text {ie }}$ is the rate at which the electrons are heated by Coulomb collisions, $Q^{\text {vis }}$ is the total rate of viscous dissipation, and $Q^{\text {rad }}$ is the radiative cooling. The parameter $\delta$ describes the fraction of the viscous heating that goes into the electrons. In general we set $\delta=10^{-3} \sim m_{e} / m_{p}$.

Because of Compton scattering, the radiative cooling term couples the flow at different radii. It is calculated using the iterative scattering method described in detail in Narayan, Barret \& McClintock (1997). The ADAF is represented by a logarithmically spaced grid of nested shells. Within each shell all flow variables are assumed uniform for $\pi / 2-\theta_{H}<\theta<$ $\pi / 2+\theta_{H}$, where $\theta_{H}$ is an effective angular scale height, calculated as in Narayan, Barret \& McClintock (1997) Appendix A. We then guess a value of the electron temperature and compute the synchrotron and bremsstrahlung emission from each shell. Then, using the probability matrix elements $P_{i j}$, which give the probability that a photon emitted in shell $i$ of the ADAF is scattered by an electron in shell $j$, the rate of cooling through 
Compton scattering is calculated (see Narayan, Barret \& McClintock 1997 for details). This procedure is iterated until convergence is achieved. The iteration method ensures that multiple scattering within the ADAF is properly taken into account. It also ensures that the advection parameter $f$ used in the dynamical solution is consistent with the actual energy advection, as determined by the radiation solution. Doppler shifts and ray deflections are ignored in the present calculations.

Once the cooling term is calculated, we must get the photons out to the observer. We use essentially Newtonian photon transport, although we have taken a step toward relativistic photon transport by including gravitational redshift (this is also included in the Compton scattering calculation). We do not include Doppler shifts, which to lowest order in $v / c$ broaden the spectrum. Since all features in our spectra have $\delta \nu / \nu$ of order unity, this should not greatly alter the gross properties of the spectrum, except where it is very steep. At next higher order in $v / c$ gravitational redshift, higher order Doppler, and various geometric effects all enter. In our model we have included a correction for the volume of the emitting region $(1 / \sqrt{1-1 / r} \times$ the Euclidean value) and for gravitational redshift. The sum of these effects is that $L_{\nu}(\nu)$ observed at large radius is now $L_{\nu}(\nu \sqrt{1-1 / r})$, that is, $L_{\nu}$ is shifted redward by the gravitational redshift factor. Jaroszynski \& Kurpiewski (1997) have recently considered the full effects of photon transport near a Kerr black hole on the spectrum of an ADAF.

The above discussion is concerned with radiation from electrons. We also compute pion production by the hot protons in the ADAF and the resulting $\gamma$-ray emission through pion decay. The procedure we follow for this calculation is described in Mahadevan et al. (1997) and is based on earlier work by Dermer (1986). The present calculations differ in three respects from those in Mahadevan et al.: 1) the density profile of the protons is now given by the relativistic global solution of Popham \& Gammie (1997) instead of the pseudoNewtonian solution of Narayan, Kato \& Honma (1997), 2) we allow for the non-spherical geometry of the flow via the angle $\theta_{H}$ mentioned above, and 3) we include the effect of gravitational redshift.

The pion production rate depends sensitively on the energy distribution of the protons. Mahadevan et al. (1997) considered two extreme distributions: a thermal distribution and a power-law distribution. The latter has significantly more pion production and maximizes the efficiency of $\gamma$-ray emission. We employ the power-law model with an energy index $p=2.3$. The choice of model is dictated by the EGRET spectrum of the Galactic Center, which is consistent with a power-law distribution of proton energies and is inconsistent with a thermal distribution (Mahadevan et al. 1997). Note that Mahadevan \& Quataert (1997) have shown that the protons in an ADAF do not have time to thermalize; therefore, 
it is permissible to assume a nonthermal energy distribution for the protons.

\section{Results}

In this section we combine the modeling techniques outlined in $\S 3$ with the observational constraints described in $\S 2$ to come up with ADAF models of Sgr A*. Our standard parameters are as follows. We assume that the ADAF extends from $r=1$ to $r_{\text {out }}=10^{5}$. For the black hole mass, we take the estimate of Eckart \& Genzel (1997): $M=2.5 \times 10^{6} M_{\odot}$. We assume exact equipartition between gas and magnetic pressure in the accreting gas: $\beta=0.5$. Following Hawley, Gammie \& Balbus (1996) we take the viscosity parameter to be $\alpha \sim 0.6(1-\beta)=0.3$ (the coefficient lies in the range $0.5-0.6$ ). We assume that electrons receive only a small fraction $\delta \sim m_{e} / m_{p}$ of the viscous dissipation as direct heating: $\delta=0.001$. We assume a Schwarzschild black hole: $a=0$. Since we do not know the orientation of the angular momentum vector of the accreting gas, we set the inclination of the system to a generic value: $i=60^{\circ}$. The models considered here are extremely optically thin and so the results are virtually independent of $i$.

The only parameter that needs discussion is $r_{\text {out }}$. In a viscous rotating accretion flow, the outer edge of the flow is determined by the radius at which the outward viscous transport of angular momentum is balanced by external torques. In the case of Sgr A* it is not clear exactly where this balance is achieved, but it is likely to be roughly at the radius where the mass supply originates. Our choice of $r_{\text {out }}=10^{5}$, corresponding to $R_{\text {out }}=0.024 \mathrm{pc}$, may be an underestimate of the true outer radius. However, this is not a serious concern for the modeling since for such large outer radii the results are insensitive to the actual value chosen. This is because the energetically important region of the flow is closer to the black hole.

As described above, all the parameters of the model are fixed at standard values in our model. The only parameter that we consider fully adjustable is the mass accretion rate $\dot{m}$. In each model described below, we have adjusted $\dot{m}$ so as to fit the ROSAT X-ray flux (but see the discussion in $\S 2$ for uncertainties in the X-ray flux due to the hydrogen column). Although we treat $\dot{m}$ as a free parameter, there are in fact some constraints. The infrared source IRS 16 appears to be the primary supplier of gas to Sgr A*. Assuming that Sgr A* captures a fraction of the wind of IRS 16 by Bondi accretion, Melia (1992) estimated $\dot{M} \approx 2 \times 10^{-4} M_{\odot} \mathrm{yr}^{-1}$ assuming a wind velocity of $600 \mathrm{~km} \mathrm{~s}^{-1}$, while Genzel et

al. (1994) estimated a mass accretion rate of $\dot{M} \approx 6 \times 10^{-6} M_{\odot} \mathrm{yr}^{-1}$ using a wind velocity of $1000 \mathrm{~km} \mathrm{~s}^{-1}$. We take these two estimates to be low and high extremes. Converting to Eddington units, $\dot{m}$ is thus likely to be in the range $10^{-4}<\dot{m}<3 \times 10^{-3}$. 
The solid line in Fig. 1 shows our baseline model where $M, \alpha, \beta$, and $\delta$ have been set to their standard values $\left(2.5 \times 10^{6} M_{\odot}, 0.3,0.5,0.001\right.$ respectively $)$ and $\dot{m}$ has been adjusted to fit the X-ray flux. This model has a mass accretion rate of $\dot{m}=1.3 \times 10^{-4}$, which lies within the acceptable range of $\dot{m}$ discussed above. The computed spectrum has four well-defined peaks. From the left, these correspond to self-absorbed thermal synchrotron emission, singly Compton-scattered synchrotron radiation, bremsstrahlung emission, and $\gamma$-rays from neutral pion decay. The model spectrum passes through the VLBI radio flux measurement at $86 \mathrm{GHz}$, which we have identified to be a reliable high frequency radio observation (cf. §2), and turns over sharply in the sub-millimeter band exactly as required by the sub-millimeter and infrared upper limits. The model also satisfies the ROSAT X-ray detection and the other X-ray upper limits. Considering that only one parameter, $\dot{m}$, has been adjusted, we consider the agreement with the data satisfactory.

Note that the infrared and X-ray fluxes have come down significantly compared to the data shown in Narayan et al. (1995). The present model is compatible with both the new measurements. Interestingly, we cannot find any model that fits either the new infrared upper limit with the old X-ray data, or the new X-ray flux with the old infrared data.

The model does not agree with the low frequency radio measurements at frequencies below $86 \mathrm{GHz}$. We discuss this discrepancy at the end of this section.

The model also has a discrepancy in the $\gamma$-ray band; the predicted flux is lower than the observations by approximately an order of magnitude. The pion decay model requires an accretion rate of $\dot{m}=4.5 \times 10^{-4}$ to fit the observed flux whereas the fit to the rest of the spectrum gives $\dot{m}=1.3 \times 10^{-4}$. There is thus a discrepancy of a factor $\sim 3-4$ between the two values of $\dot{m}$. We have been unable to come up with a reasonable resolution of this discrepancy. Considering that we have used an extreme model which maximizes the pion production rate (cf. §2), we are compelled to suggest that perhaps the EGRET source does not correspond to Sgr $\mathrm{A}^{*}$, but rather to a concentration of molecular gas at the Galactic Center. The resulting emission is then expected to be much more diffuse, but it would still appear unresolved to the $1^{\circ}$ beam of EGRET.

Two features of the ADAF model in Fig. 1 should be highlighted. First, the model fits the data using a reasonable mass for the black hole, $M=2.5 \times 10^{6} M_{\odot}$. This is an improvement over the model described in Narayan et al. (1995) where the data could be fitted only with a mass $M=7 \times 10^{5} M_{\odot}$. The primary reason for the improvement is the inclusion of electron energy advection, or compressive heating of the electrons (Nakamura et al. 1997), as we explain below. Second, the model is extremely advection-dominated. The bolometric luminosity $L_{\mathrm{bol}}$ integrated over all frequencies is only $2.1 \times 10^{36} \mathrm{erg} \mathrm{s}^{-1}$, which corresponds to a radiative efficiency, $\epsilon=L_{\mathrm{bol}} / \dot{M} c^{2}=5 \times 10^{-6}$. It is this extraordinarily 
low radiative efficiency that allows the model to fit the observations with such a large mass accretion rate.

In contrast, a standard thin accretion disk model of $\mathrm{Sgr} \mathrm{A}^{*}$ is ruled out quite comprehensively by the radio-IR-X-ray data. The dotted line in Fig. 1 shows a thin disk model with $\dot{m}=10^{-4}$, the lowest mass accretion rate that we consider reasonable. The spectrum was calculated assuming that the emission is blackbody at each radius. The model predicts an infrared flux which is many orders of magnitude above the measured upper limits. The short dashed line in Fig. 1 shows another thin disk model where $\dot{m}$ has been reduced to $10^{-9}$. This model does satisfy the IR upper limits but does not fit any of the observations, either in the radio or X-ray bands. Further, the mass accretion rate is unreasonably small.

A number of improvements have taken place in the modeling techniques since the publication of our first model of Sgr A* (Narayan et al. 1995). It is interesting to investigate what effect each improvement has had on the calculated spectrum. Figure 2 shows a sequence of models in which we start with the simplest version of the model and progressively add features one by one. Figures 3-5 explore the effects of varying model parameters and are described below.

The dotted line in Fig. 2a corresponds to the most primitive version of the ADAF model, in which the flow is assumed to have a self-similar form (Narayan \& Yi 1994) and neither compressive heating of electrons nor gravitational redshift is included. The optimized accretion rate is $\dot{m}=6 \times 10^{-5}$. The general shape of the spectrum is similar to that of our standard model (the solid line), but this model differs in three ways. First, the emission is stronger in nearly all bands compared to our standard model; in fact, the model is inconsistent with the infrared limit. Second, the mass accretion rate is lower than in our standard model by a factor of 2 . This, combined with the higher luminosity, means that the model is not as advection-dominated as our standard model; we obtain a radiative efficiency of $\epsilon=2.4 \times 10^{-4}$. Third, the calculated synchrotron emission is well above the 86 $\mathrm{GHz}$ VLBI point. The discrepancy in the radio flux is more than an order of magnitude and is serious. Within the framework of the self-similar flow assumption, the only way to eliminate this problem is by changing one or more of the model parameters. This was, in fact, the primary reason why the Narayan et al. (1995) model required a black hole mass of $7 \times 10^{5} M_{\odot}$ instead of the current best estimate of $2.5 \times 10^{6} M_{\odot}$ (see Fig. 3 below which shows how the spectrum is modified when the black hole mass is reduced).

The short-dashed line in Fig. 2a shows the effect of replacing the self-similar flow by a global flow based on a pseudo-Newtonian potential (Narayan, Kato \& Honma 1997; Chen, Abramowicz \& Lasota 1997). The changes are minimal. 
The long-dashed line in Fig. 2a next shows the effect of including compressive heating (Appendix A and Nakamura et al. 1997). This model, which has $\dot{m}=1.1 \times 10^{-4}$, has a very different spectrum compared to the previous two models. The overall emission is significantly reduced, notably in the synchrotron peak, and the flow is substantially more advection-dominated: $\epsilon=2.1 \times 10^{-6}$. This model fits the $86 \mathrm{GHz}$ data point very well without requiring any adjustment to the black hole mass. We thus confirm the result of Nakamura et al. (1997) that compressive heating is an important effect. Figure 2b shows the electron temperature profiles of the various models. The model with compressive heating has a significantly different temperature structure than the models without. At very low $\dot{m}$, as in our model of Sgr $\mathrm{A}^{*}$, the dominant terms in the electron energy equation are the two pieces of $Q^{\text {eadv }}$ written in the Appendix, viz. the terms proportional to $d T_{e} / d R$ and $d \rho / d R$. These two dominant terms balance each other, while the rest of the terms in equation (1) are small. In other words, the electrons are essentially adiabatic. The adiabatic condition gives a lower electron temperature than in the previous two models and this accounts for the change in the shape of the synchrotron contribution to the spectrum.

The dash-dot line in Fig. 2a shows next the effect of including a fully relativistic global solution $\left(\dot{m}=1.3 \times 10^{-4}\right)$, taken from Popham \& Gammie (1997), instead of the pseudoNewtonian global solution employed in the previous two models. Most of the features are similar, but the overall emission is increased because the relativistic solution has a lower radial velocity close to the black hole compared to the pseudo-Newtonian solution. (This is because $v<c$ at all radii in the relativistic model whereas the pseudo-Newtonian model gives $v>c$ close to the black hole.) Consequently, the density is higher in the relativistic model and this leads to increased emission.

Finally, the solid line in Fig. 2a shows our standard model, which includes gravitational redshift. As expected, this model has a lower luminosity than the previous model, but is otherwise quite similar.

Figures 3-5 show the effect of varying the other parameters in the model. Figure $3 \mathrm{a}$ shows what happens when we vary the mass. In addition to the baseline model, we present two other models with $M=1 / 3$ and 3 times $M$ of the baseline model. As Mahadevan (1997) has shown, with increasing $M$, the magnetic field strength decreases as $M^{-1 / 2}$ and this causes the synchrotron peak to move to lower frequencies $\propto M^{-1 / 2}$. Of the three models shown in Fig. 3a, the one with $M=2.5 \times 10^{6} M_{\odot}$ gives the best fit to the VLBI radio data. Assuming our choices of $\alpha$ and $\beta$ are correct, this provides additional support for the black hole mass measured by Haller et al. (1996) and Eckart \& Genzel (1997).

Figure $3 \mathrm{~b}$ shows the effect of varying the mass accretion rate. In addition to the baseline model, four other models are shown, with $\dot{m}=1 / 2,1 / \sqrt{2}, \sqrt{2}$, and 2 times $\dot{m}$ 
of the baseline model. The radiative efficiency of ADAFs varies rapidly with $\dot{m}: \epsilon \propto \dot{m}$ (Narayan \& Yi 1995b). Therefore, the luminosity varies as $\dot{m}^{2}$. This can be seen in both the synchrotron and bremsstrahlung peaks. The Compton peak in the infrared shows an even stronger dependence on $\dot{m}$. This is because the Comptonized flux is proportional to the product of the synchrotron emission (which is $\propto \dot{m}^{2}$ ) and the optical depth (which is $\propto \dot{m}$ ), so that the amplitude of this peak varies approximately as $\dot{m}^{3}$. The three intermediate models in Fig. 3b are consistent with all the data, but the two extreme models lie outside the X-ray error box and one of them also violates the sub-millimeter and infrared limits. Since the X-ray luminosity of Sgr $\mathrm{A}^{*}$ is uncertain, Fig. 3b indicates how the model $\dot{m}$ will need to be modified if a future determination of $N_{H}$ leads to a significant revision of the X-ray flux.

Figure 4a shows the effect of varying $\alpha$. For each $\alpha$, we have adjusted the accretion rate to fit the X-ray flux. The spectrum is not very sensitive to $\alpha$ (except in the optical/IR), though the fitted mass accretion rates show modest variations: $\dot{m}=6.3 \times 10^{-4}, 1.0 \times$ $10^{-3}, 1.3 \times 10^{-3}, 1.5 \times 10^{-3}$ for $\alpha=0.1,0.2,0.3,0.4$ respectively.

Figure $4 \mathrm{~b}$ shows the effect of varying $\beta$. Again, for each $\beta$, we have adjusted the accretion rate to fit the $\mathrm{X}$-ray flux. In this case, we see quite substantial changes in the predicted spectrum. An increase in $\beta$ leads to a decrease in the magnetic field strength and one may be tempted to think that this would cause the synchrotron peak to reduce in amplitude. In fact, the opposite behavior is seen. The reason can be traced to compressive heating. Since the electrons are effectively adiabatic, their temperature profile is determined by their adiabatic index $\gamma_{e}$. We show in Appendix A that because the gas is a mixture of particles and magnetic field, the effective $\gamma_{e}$ depends on the parameter $\beta$. As $\beta$ goes up, the magnetic pressure goes down and $\gamma_{e}$ increases. A larger $\gamma_{e}$ leads to hotter electrons, see Fig. 4c, which causes more synchrotron emission. Unfortunately, this means that the results are sensitive to the details of how we model energy advection in the electrons. In this context we note that our equation of state for the electrons differs somewhat from the one used by Nakamura et al. (1997). We use a relativistic equation of state which causes $\gamma_{e}$ to change as the electron temperature approaches and crosses the value $T_{e} \sim m_{e} c^{2} / k$. In addition, we modify the adiabatic index of the electrons to allow for the equipartition magnetic field which is assumed to be present in the gas and is coupled to the electrons. Nakamura et al. (1997) treat the particles as a separate component and ignore the field in their equation of state.

Figure $4 \mathrm{~d}$ shows the effect of varying $\delta$. For all $\delta \lesssim 0.01$ the spectrum is generally unaffected. This result is different from that found in Narayan, Barret \& McClintock (1997). Once again, the reason is the inclusion of compressive heating. Because the electrons are 
now essentially adiabatic, their temperature profile is not affected by modest changes in the heating or cooling. Only when $\delta$ is large, e.g. $\delta=0.03162$ (the dot-dashed line in Fig. 4d), do the electrons experience significant additional heating and only then does the spectrum show a noticeable change.

We turn now to the low frequency radio data, where the model deviates substantially from the measurements. As discussed in $\S 2$, this region of the spectrum may possibly arise from a separate component such as a jet which is outside the scope of our model. The problem with the jet proposal is that the additional component has to be quite compact $(\$ 15 \mathrm{AU})$ in order not to be seen in the high resolution VLBI images of Marcaide et al. (1992) and Alberdi et al. (1993) at $22 \mathrm{GHz}$. If the low frequency radio emission is from the accretion flow itself, then we need to identify a mechanism which could increase the radio emission over and above our baseline model.

In our model, the synchrotron radiation at different frequencies are produced at different radii in the flow, the emission at $10^{12} \mathrm{~Hz}$ coming from close to the black hole and the emission at lower frequencies coming from farther out. Thus, one simple way of improving the fit to the radio data is to modify the electron temperature outside of a few tens of Schwarzschild radii. Figure 5 is only an illustration. The solid line in Fig. 5a corresponds to our baseline model, while the dashed line is another model which is identical in all respects except that we have arbitrarily set $T_{e}=2 \times 10^{9} \mathrm{~K}$ over the radius range $r=20-1000$ (Fig. 5b). This ad hoc model fits the data well.

Is there any reason to think that the electrons might have the profile shown in Fig. 5b? The answer is tentatively yes, since there are several radial transport mechanisms which could drive the electrons to a nearly isothermal state. First, the long mean free path of the electrons can lead to fairly strong radial heat conduction (parallel to field lines). Second, synchrotron radiation, which has a thermalizing effect on the electrons (Mahadevan \& Quataert 1997), can also cause significant energy diffusion. Finally, the tangled magnetic fields which we assume to be present in the flow may move outwards as a result of buoyancy and may dissipate their energy and heat the electrons at larger radii. Some of these effects could well be episodic; it is interesting in this connection that the radio flux of Sgr A* is known to be quite variable (Zhao et al. 1989). These effects need further study.

\section{Discussion}

The main result of this paper is that the two-temperature ADAF model (Narayan et al. 1995, Rees 1982) provides a viable explanation of the spectral properties of Sgr 
A* $^{*}$ Our basic model, shown by the solid line in Fig. 1, fits most of the high resolution measurements from the high frequency $(\geq 86 \mathrm{GHz})$ radio to the X-ray band, including the stringent infrared limit of Menten et al. (1997) and the revised X-ray luminosity.

The ADAF model described in this paper makes use of a two-temperature plasma, as originally described by Shapiro et al. (1976). This involves two key assumptions. First, it is assumed that most of the energy released by viscosity goes into the ions and not the electrons. Second, the only coupling between ions and electrons is assumed to be via Coulomb collisions. At the present time, these assumptions have not been proved theoretically to be valid, nor have they been disproved (e.g. Begelman \& Chuieh 1988). Rees et al. (1982) made the interesting point that the plasma physics involved is so complex that perhaps the best way of testing the two-temperature paradigm is by comparison of astrophysical models with observations. Fabian \& Rees (1995) declared, based on the success of our earlier model of Sgr A* (Narayan et al. 1995), that the two-temperature assumption appears to be supported by observations. As the present paper shows, the two-temperature ADAF model explains the data on Sgr A* even better than it seemed in the 1995 paper. Furthermore, the ADAF model has been successfully applied to several other sources (see Narayan 1997 for a review), making the Fabian \& Rees argument even stronger.

Although the ADAF model presented here includes a number of parameters, only one parameter is adjusted in the spectral fits, and the quality of the agreement between the model and the data in Fig. 1 must be judged in this context. The free parameter in the model is the Eddington-scaled mass accretion rate $\dot{m}$ which is adjusted so as to fit the X-ray luminosity in the ROSAT band. Although there is some uncertainty in the X-ray luminosity because of uncertainty in the hydrogen column (cf. §2), we feel this does not seriously affect the model fit (see Fig. 4c which shows how small adjustments in the fitted parameter $\dot{m}$ will allow the model to remain consistent with any future revisions of the X-ray luminosity).

Apart from $\dot{m}$ all the other parameters in the model are assigned standard values (these values are not changed from one application to another). For instance, we invoke strict equipartition and assign $\beta=0.5$. Once $\beta$ is given, the model has a unique prescription (Esin 1997) for the adiabatic index of the gas: $\gamma=(8-3 \beta) /(6-3 \beta)$. Further, by making use of the scaling between the shear stress and the magnetic pressure derived by Hawley, Gammie \& Balbus (1996), we are also able to fix the viscosity parameter: $\alpha \sim 0.6(1-\beta)$. Of course, the use of the $\alpha$ prescription itself involves an assumption about the nature of the viscosity. Narayan (1996), however, has argued that this viscosity prescription with $\alpha$ independent of radius is particularly appropriate for ADAFs. The final parameter in the model is $\delta$, the fraction of the viscous energy that goes directly into the electrons. By 
assumption $\delta$ is small, and it is usually assigned a value $\delta=10^{-3} \sim m_{e} / m_{p}$. The results are, however, insensitive to the exact value so long as $\delta \lesssim 10^{-2}$ (see Fig. 4d).

The models presented here are substantially more elaborate (cf. §2) than the ones described in Narayan et al. (1995). It is pleasing that the more detailed analysis presented here leads to an improvement in the agreement with observational constraints. In contrast to the models presented in Narayan et al. (1995), where a black hole mass of $7 \times 10^{5} M_{\odot}$ was required in order to accommodate the radio data, here we find that the model quite naturally fits the data with a more reasonable mass of $2.5 \times 10^{6} M_{\odot}$ (Eckart \& Genzel 1997). This removes one of the main problems with the previous work. The model also predicts a mass accretion rate $\dot{m} \sim 1.3 \times 10^{-4}$ (in Eddington units) which is within the range considered likely by a direct estimate of the accretion rate (Genzel et al. 1994).

We should emphasize that the model is self-consistent, and includes a detailed treatment of hydrodynamics, radiation processes, and thermal balance of ions and electrons in the twotemperature plasma. Most other models in the literature are more primitive and/or ad hoc. In addition, the model is fairly robust to changes in the parameters (Fig. 4). The predicted spectrum is insensitive to large variations in the viscosity parameter $\alpha$ and the electron heating parameter $\delta$. The results do, however, vary with the equipartition parameter $\beta$ (Fig. 4b, 4c).

There are some problems with the present model. First, we under-predict the $\gamma$-ray flux in the EGRET band by an order of magnitude. This might indicate a residual uncertainty in the relative normalization of the protons and electrons, but we have not been able to come up with any specific proposal to eliminate the discrepancy. It is worrying that we underpredict the flux even though we have assumed a power-law energy distribution for the protons which maximizes the predicted $\gamma$-ray luminosity. Perhaps the $\gamma$-ray source detected by EGRET with its one degree beam is not Sgr $\mathrm{A}^{*}$ at all, but diffuse emission from a molecular cloud in the Galactic Center region.

Another problem is that the model under-predicts the radio flux below $86 \mathrm{GHz}$. The existence of a break in the radio spectrum at around $86 \mathrm{GHz}$ suggests that the region of the spectrum below $86 \mathrm{GHz}$ might arise from a distinct component, e.g. a weak outflow of some kind, as postulated in the model due to Falcke (1996). We note in this connection that Krichbaum et al. (1993) claim to have seen evidence for a jet in their $43 \mathrm{GHz}$ VLBI image, though Backer et al. 1993 could not verify this. One problem with the jet hypothesis is that the $22 \mathrm{GHz}$ VLBI maps of Marcaide et al. (1992) and Alberdi et al. (1993) show no evidence for any resolved structure. This means that the jet has to be smaller than $15 \mathrm{AU}$, i.e. no larger than ten times the size we would predict for the ADAF at this frequency. If the radio flux is from a jet, then clearly the discrepancy we face in the radio luminosity is 
not an issue since our model includes only the accretion flow. However, if the radiation is not from a separate component but from the ADAF itself, then it probably implies that the electron temperature in the flow differs from that predicted by our model. Figure 5 shows the kind of temperature profile that is needed to fit the observations. The profile shown here is completely ad hoc. However, if radial transport processes are included, the model might give a qualitatively similar temperature profile. We would emphasize that the luminosity of $\operatorname{Sgr} \mathrm{A}^{*}$ in low frequency radio waves is a small fraction (less than a percent) of the bolometric luminosity of the source. Minor changes in the flow profile can explain the discrepancy here without affecting the overall flow dynamics or energetics.

Yet another problem is the somewhat low electron brightness temperatures predicted by the model. As Fig. 4c shows, we obtain values below $10^{10} \mathrm{~K}$ at all radii, whereas VLBI observations at $43 \mathrm{GHz}$ and $86 \mathrm{GHz}$ indicate brightness temperatures in excess of $10^{10}$ $\mathrm{K}$ (Backer et al. 1993, Rogers et al. 1994). Thus, although the model fits the $86 \mathrm{GHz}$ flux well, it seems to predict a larger angular size for the source, by a factor of $\sim 1.5$, than observations indicate. For comparison with future observations, we list here linear source radii at various radio frequencies according to the baseline model shown in Fig. 1: $1.3 \times 10^{12} \mathrm{~cm}$ at $10^{12.5} \mathrm{~Hz}, 4.1 \times 10^{12} \mathrm{~cm}$ at $10^{12} \mathrm{~Hz}, 1.0 \times 10^{13} \mathrm{~cm}$ at $10^{11.5} \mathrm{~Hz}, 2.1 \times 10^{13}$ $\mathrm{cm}$ at $10^{11} \mathrm{~Hz}, 4.1 \times 10^{13} \mathrm{~cm}$ at $10^{10.5} \mathrm{~Hz}, 8.3 \times 10^{13} \mathrm{~cm}$ at $10^{10} \mathrm{~Hz}$. Note that the electron temperature depends on the value of the parameter $\beta$ (Fig. 4c). Thus, one might be able to fit the observed brightness temperatures by tuning this parameter as a function of radius, although we have kept $\beta$ fixed in our models.

An outstanding feature of the ADAF model presented here is its extraordinarily low radiative efficiency: $\epsilon=L_{\mathrm{bol}} / \dot{M} c^{2}=5 \times 10^{-6}$. The low efficiency permits the model to fit the very low luminosity of $\operatorname{Sgr} A^{*}$ with a fairly large $\dot{m}$. Figure 1 shows two thin disk models with the standard radiative efficiency of $\epsilon \sim 0.1$. A thin disk with the "correct" $\dot{m}$ (dotted line) over-predicts the infrared flux by four or five orders of magnitude. A model with $\dot{m}$ reduced by a factor of $10^{5}$ accommodates the IR limits but does not fit any of the other data and has a mass accretion rate which is extremely discrepant with independent estimates of $\dot{m}$. Some models in the literature which are based on a thin disk attempt to solve the luminosity problem by hiding a large fraction of the emission in the optical band where there is extinction by dust (e.g. Falcke et al. 1993a, Melia 1994). However, the models invariably predict emission in the infrared at a level well above the Menten et al. (1997) limit. Other models in the literature which are not based on a thin disk also have problems fitting the low luminosity (e.g. Duschl \& Lesch 1994; Mastichiadis \& Ozernoy 1994) and generally require values of $\dot{m}$ significantly below the lower limit of $10^{-4}$.

Can we save the thin disk model by assuming that the disk has no viscosity at all, 
so that the gas in the disk does not accrete at the present time? In a very interesting argument, Falcke \& Melia (1997) show that even such an extreme model is inconsistent. This is because the mere presence of the disk, even though it does not accrete, will still lead to fairly strong infrared emission. The argument is that if there is inflow of material towards the Galactic Center via a Bondi-Hoyle-like flow, when the inflowing gas hits the disk and circularizes at a radius $r_{\text {circ }}$ it will produce a substantial amount of thermal radiation. For the mass accretion rate of $\dot{m} \gtrsim 10^{-4}$ estimated in the case of Sgr A* (cf. $\S 4$ ) the predicted infrared flux is well above the Menten et al. (1997) limit in nearly all the cases considered by Falcke \& Melia (1997). The only way of avoiding this argument is by making $r_{\text {circ }}$ large (say $r_{\text {circ }} \gtrsim 10^{4}$ ), but Falcke \& Melia show that this is not consistent with most reasonable wind specific angular momentum configurations.

The ADAF model is able to circumvent the Falcke \& Melia argument quite naturally and this is another point in favor of this model. Since the accreting gas in the ADAF has a quasi-spherical shape (Narayan \& Yi 1995a), the incoming Bondi-Hoyle flow never reaches $r_{\text {circ }}$. Instead it shocks with the ADAF at a distance on the order of the outer radius $R_{\text {out }}$ (recall that $H \sim R$ in an $\mathrm{ADAF}$ ). In our models we have taken $R_{\text {out }}=10^{5} R_{s}$, but as we argued in $\S 3$ the outer radius may be even larger. The luminosity associated with the impact at such large radii is quite low. Once the stream has been incorporated into the $\mathrm{ADAF}$ at the outer radius, the accretion switches to an advection-dominated form and the gas does not radiate very much as it moves in.

Several recent papers have made the point that the successful application of the ADAF model to any observed system is direct evidence that the accreting object is a black hole (Narayan, McClintock \& Yi 1996; Narayan, Yi \& Mahadevan 1996; Narayan, Barret \& McClintock 1997; Narayan, Garcia \& McClintock 1997). The argument is that if accretion is via an ADAF and if the object has an event horizon, then the advected energy will disappear from sight. However, if the central object has a surface, then the surface will be heated by the hot inflow from the ADAF and the advected thermal energy will be emitted as thermal radiation. Thus, for an object with a surface, the radiative efficiency will be the canonical $10 \%$ even if the accretion occurs via an ADAF. Only if the central object is a black hole can the radiative efficiency be truly low.

Sgr $A^{*}$ is perhaps one of the best objects for this argument. This source provides two strong observational constraints: (1) The mass accretion rate is estimated to be at least a few $\times 10^{-6} M_{\odot} \mathrm{yr}^{-1}$, and (2) the bolometric luminosity is no greater than $10^{37} \mathrm{erg} \mathrm{s}^{-1}$. In our opinion the only plausible resolution of these two conflicting pieces of evidence is to postulate (1) that the accretion in Sgr $\mathrm{A}^{*}$ occurs via an ADAF and (2) that the central object is a supermassive black hole. This "proof" of the black hole nature of Sgr A* is 
qualitatively different from usual proofs which rely on a measurement of the mass. The usual argument is that if an object is too massive to be a neutron star it must be a black hole; it is a proof by elimination. Our "proof" is somewhat more direct and cuts to the essence of what constitutes a black hole, namely the presence of an event horizon. We argue that Sgr $\mathrm{A}^{*}$ has an enormous luminosity deficit for plausible values of $\dot{M}$ and the simplest explanation is that the object has an event horizon.

One of the results to come out of this work is our confirmation that compressive heating of electrons cannot be ignored and must be included consistently in computations of ADAF spectra of low luminosity systems like Sgr A* (see Fig. 2). This point was made recently in an important paper by Nakamura et al. (1997). In view of this result it would be useful to revisit other low- $\dot{m}$ systems to which the ADAF model has been applied (e.g. soft X-ray transients, Narayan, Barret \& McClintock 1997; Hameury et al. 1997; NGC 4258, Lasota et al. 1996; low-luminosity nuclei of giant ellipticals, Fabian \& Rees 1995; Mahadevan 1997; Reynolds et al. 1996) and redo the analysis with the inclusion of compressive heating. Interestingly, Esin et al. (1997) have shown that compressive heating has a much weaker effect on ADAFs at higher values of $\dot{m}$. The reason is that with increasing $\dot{m}$ the other terms in the electron energy equation (1), notably the Coulomb collision term $Q^{\text {ie }}$ and the radiative cooling term $Q^{\text {rad }}$, become more important, and compressive heating no longer dominates.

In the model presented in this paper the emission in the infrared and the radio (above about $86 \mathrm{GHz}$ ) comes from fairly small radii $\sim 10 R_{s}$. Since the gas in the ADAF is nearly in free-fall, the characteristic time scales of the flow are quite short. We may thus expect rapid variability in Sgr $\mathrm{A}^{*}$ at these wavelengths. The shortest likely time scale is the dynamical time, $t_{\mathrm{dyn}} \sim\left(G M / R^{3}\right)^{-1 / 2}$, which gives $t_{\mathrm{dyn}} \sim 5000 \mathrm{~s}$ at $R=10 R_{s}$. The viscous time is longer than this by a factor of $1 / \alpha \sim 3$. The longer wavelength radio emission is from larger radii; for instance, the emission below $1 \mathrm{GHz}$ comes from $R \gtrsim 10^{3} R_{s}$. Variability at these wavelengths will be correspondingly slower: $t_{\mathrm{dyn}}\left(10^{3} R_{s}\right) \sim 5 \times 10^{6} \mathrm{~s}$. The bremsstrahlung emission in soft $\mathrm{X}$-rays is from a broad range of radii, but is dominated by large radii. Therefore, the soft X-ray flux should show much slower variations (timescale $\sim 1$ year) than the radio, millimeter or infrared fluxes. However, the hard X-ray $(\sim 100 \mathrm{keV})$ flux at the broad peak of the $\nu L_{\nu}$ spectrum will trace the higher temperature $\left(\sim 10^{9}-10^{10} \mathrm{~K}\right)$ inner regions and could vary on time scales of days.

By assumption, the electrons in our model are fully thermal. Mahadevan \& Quataert (1997) have, however, shown that at low mass accretion rates similar to that in Sgr A* , electrons may not be thermalized at larger radii. The electron energy distribution at these radii may then be truncated even more sharply than in a Maxwellian, which will cause a 
reduction in the synchrotron emission. This will act to increase the discrepancy between the model and the data at low radio frequencies. This is an area for further work.

Note, however, that a power-law distribution of electrons extending over any reasonable range of energies is constrained by the observations. For instance, since optically thin nonthermal synchrotron emission usually has a spectral form $L_{\nu} \sim \nu^{-0.7}$, this means that in a $\nu L_{\nu}$ plot the optically thin emission would continue to rise as $\nu^{+0.3}$. Such a rise beyond $\sim 10^{12}-10^{13} \mathrm{~Hz}$ is ruled out by the observed upper limits in the infrared.

In this connection, we note that Falcke (1996), Duschl \& Lesch (1994) and Beckert \& Duschl (1997) have proposed nonthermal models for Sgr A* in which most of the electrons have Lorentz factors of around a few hundred. These authors require a mono-energetic electron distribution in order to reproduce the sharp cutoff observed in the sub-millimeter/FIR band. Falcke assumes a mono-energetic distribution in the "nozzle" of his jet-disk model, while Duschl \& Lesch assume a homogeneous sphere of mono-energetic electrons. Such mono-energetic models are not ruled out by the argument of the previous paragraph and indeed both groups are able to fit the radio spectrum of Sgr A* reasonably well, although the sub-mm, IR and X-ray spectrum are not explained. We, however, find the idea of a nonthermal but mono-energetic distribution somewhat artificial. Our ADAF model fits the data in a more natural way by making use of a thermal distribution of electrons. Further, because of thermal synchrotron self-absorption, the cutoff of the spectrum in the sub-millimeter band is very sharp without requiring any fine-tuning. Melia's (1992, 1994) model also makes use of self-absorbed thermal synchrotron emission and has similar properties, but there is an error in his calculation of the synchrotron emissivity (cf. Mahadevan, Narayan \& Yi 1996).

Further high spatial resolution observations of Sgr A* are highly desirable. The ADAF model can be tested and constrained with better observations of the spectrum and variability, especially in the millimeter, sub-millimeter, infrared and X-ray bands. Better $\gamma$-ray observations might also resolve the issue of whether the source detected by EGRET is Sgr $\mathrm{A}^{*}$.

After this paper was submitted we saw a closely related preprint by Manmoto, Mineshige \& Kusunose (1997) entitled "Spectrum of optically thin advection dominated accretion flow around a black hole: application to Sgr A*." There are many points of similarity between their paper and ours and fairly close agreement in the results. In particular, Manmoto et al. emphasize the importance of the advection term in the heating of electrons. The main difference between the papers is in the choice of equation of state of the electrons. We allow for the effect of magnetic fields in calculating the adiabatic index of the electrons and we also include relativistic corrections (see Appendix A) whereas Manmoto et al. em- 
ploy a simpler prescription. Also, we make use of a more comprehensive and updated set of spectral data for the model comparisons.

This work was supported in part by NSF grant AST 9423209 and NASA grant NAG 52837. We thank Shoji Kato for sending us preprints of Nakamura et al. (1997) and Manmoto et al. (1997) prior to publication, and T. Krichbaum, J. Marcaide, S. Stolovy and E. Serabyn for comments on the spectral data. RM thanks Jun-Hui Zhao and Mark Reid for useful discussions on the radio observations of the Galactic Center. 


\section{A. Energy Advection by Electrons}

The advection term in equation (1) can be written per unit volume as

$$
Q^{\mathrm{e}, \mathrm{adv}}=\rho T_{e} v \frac{d s_{e}}{d R}
$$

where $s_{e}$ is the entropy of the electrons per unit total gas mass. This term was ignored in much of the earlier work, on the assumption that the temperature of the electrons is determined primarily by a balance between the Coulomb transfer term $Q^{\text {ie }}$ and the radiative cooling term $Q^{\text {rad }}$ in equation (1). However, Nakamura et al. (1997) have shown that energy advection by electrons can be important under some circumstances and may even play a dominant role in determining the electron temperature. This is the case especially when the mass accretion rate is low, as in Sgr $\mathrm{A}^{*}$.

We begin with the relation

$$
T_{e} d s_{e}=d u_{e}+P_{e} d\left(\frac{1}{\rho}\right)
$$

where $u_{e}$ is the internal energy of the electrons per unit mass and $P_{e}$ is the electron pressure. We consider a mixture of gas and magnetic fields. If gas pressure contributes a constant fraction $\beta$ to the total pressure $P_{t o t}$, then

$$
P_{t o t}=\frac{\rho k T_{i}}{\mu_{i} m_{u}}+\frac{\rho k T_{e}}{\mu_{e} m_{u}}+\frac{B^{2}}{24 \pi}=\frac{\rho k T_{i}}{\beta \mu_{i} m_{u}}+\frac{\rho k T_{e}}{\beta \mu_{e} m_{u}} .
$$

It seems natural to denote the two terms on the right as the effective ion and electron

pressure, each including an appropriate fraction of the magnetic pressure. Therefore, we have $P_{e}=\rho k T_{e} /\left(\beta \mu_{e} m_{u}\right)$.

The internal energy of the gas is a sum of the ion, electron and magnetic field internal energies:

$$
u=\frac{3}{2} \frac{k T_{i}}{\mu_{i} m_{u}}+a\left(T_{e}\right) \frac{k T_{e}}{\mu_{e} m_{u}}+\frac{B^{2}}{8 \pi \rho}=\frac{6-3 \beta}{2 \beta} \frac{k T_{i}}{\mu_{i} m_{u}}+\left[\frac{3(1-\beta)}{\beta}+a\left(T_{e}\right)\right] \frac{k T_{e}}{\mu_{e} m_{u}},
$$

where the coefficient $a\left(T_{e}\right)$ varies from $3 / 2$ in the case of a non-relativistic electron gas, to 3 for fully relativistic electrons. The general expression for $a$ as a function of the dimensionless electron temperature $\theta_{e}=k T_{e} / m_{e} c^{2}$ was derived by Chandrasekhar (1939, Chapter X, eq. $[236])$ :

$$
a\left(\theta_{e}\right)=\frac{1}{\theta_{e}}\left(\frac{3 K_{3}\left(1 / \theta_{e}\right)+K_{1}\left(1 / \theta_{e}\right)}{4 K_{2}\left(1 / \theta_{e}\right)}-1\right) .
$$


Note that the ions never become relativistic in these flows, so that the corresponding coefficient for the ions is always $\sim 3 / 2$.

As we have done for the pressure, the right hand side of Eq. (A4) may be naturally divided into two terms, the ion and electron internal energies:

$$
u_{i}=\frac{6-3 \beta}{2 \beta} \frac{k T_{i}}{\mu_{i} m_{u}}, \quad \text { and } \quad u_{e}=\left[\frac{3(1-\beta)}{\beta}+a\left(T_{e}\right)\right] \frac{k T_{e}}{\mu_{e} m_{u}} .
$$

In this interpretation, $u_{i}$ and $u_{e}$ are again "effective" quantities, which include contributions from the particles as well as the associated magnetic field. Note that the contribution of the magnetic field to the internal energy of each particle species is proportional to the contribution of these particles to the total pressure, a natural choice in our model where the ratio of the magnetic to gas pressure is fixed. Having defined $P_{e}$ and $u_{e}$, we can now write the energy advection term for the electrons as

$$
Q^{\mathrm{e}, \mathrm{adv}}=\frac{\rho v k}{\mu_{e} m_{u}}\left[\frac{3(1-\beta)}{\beta}+a\left(T_{e}\right)+T_{e} \frac{d a}{d T_{e}}\right] \frac{d T_{e}}{d R}-\frac{v k T_{e}}{\beta \mu_{e} m_{u}} \frac{d \rho}{d R} .
$$

¿From the pressure and internal energy of the electrons, we calculate the effective adiabatic index of the electrons via the relation $\gamma_{e}-1=P_{e} / u_{e}$. This gives

$$
\gamma_{e}=\frac{4-3 \beta+a \beta}{3-3 \beta+a \beta} .
$$

If the particles are relativistic, then $a=3$ and $\gamma_{e}=4 / 3$ regardless of $\beta$. This is because both the particles and the tangled field behave like radiation. If the particles are non-relativistic, however, $a=3 / 2$ and in this case

$$
\gamma_{e}=\frac{8-3 \beta}{6-3 \beta},
$$

as shown by Esin (1997). We see that $\gamma_{e}$ varies from $4 / 3$ when $\beta=0$ to $5 / 3$ when $\beta=1$. Thus, the adiabatic index depends fairly sensitively on $\beta$ so long as the electrons are nonrelativistic. If the electrons in the accreting gas behave adiabatically, then their temperature varies as $T_{e} \propto \rho^{\gamma_{e}-1}$ (technically there is also the term proportional to $d a / d T_{e}$ in eq A7 which becomes important when the electrons are quasi-relativistic, but we neglect this for simplicity). Thus, for a given amount of compression, the electrons are hotter when $\beta$ is large and cooler when $\beta$ is small. This explains the trend seen in Figs. $4 \mathrm{~b}$ and $4 \mathrm{c}$.

\section{REFERENCES}

Abramowicz, M.A., Chen, X., Granath, M., \& Lasota, J.-P. 1996, ApJ, 471, 762 
Abramowicz, M.A., Chen, X., Kato, S., Lasota, J.-P., \& Regev, O. 1995, ApJ, 438, L37

Abramowicz, M.A., Lanza, A., \& Percival, M.J. 1997, ApJ 479, 179

Alberdi, A., et al., 1993, A\&A, 277, L1

Backer, D., C, 1982, in Proc. IAU Symposium, eds. D.S. Heeschen \& C. M. Wade, 97, 389

Balick, B., \& Brown, R., 1974, ApJ, 194,265

Barret, D., \& Grindlay, J. E., 1995, ApJ, 440, 841

Beckert, T., \& Duschl, W. J. 1997, A\&A, in press (astro-ph/9707162)

Beckert, T., Duschl, W. J., Mezger, P. G., \& Zylka, R., 1996, A\&A, 307, 450

Begelman, M. C., \& Chuieh, T. 1988, ApJ, 332, 872

Brown, R. L, \& Lo, K. Y., 1982, ApJ, 253, 10

Chandrasekhar, S., 1939, in Introduction to the Study of Stellar Structure, (New York: Dover Publications)

Chen, X., Abramowicz, M. A., \& Lasota, J.-P. 1997, ApJ, 476, 61

Chen, X., Abramowicz, M. A., Lasota, J.-P., Narayan, R., \& Yi, I. 1995, ApJ, 443, L61

Davies, R. D., Walsh, D., \& Booth, R. S., 1976, MNRAS, 177, 319

Dent, W. R. F., Matthews, H. E., Wade, R., \& Duncan, W. D., 1993, ApJ, 410, 650

Dermer, C. D. 1986, A\&A, 157, 223

Dermer, C. D., Liang, E. P., \& Canfield, E. 1991, ApJ, 369, 410

Duschl, W. J., \& Lesch, H. 1994, A\&A, 286, 431

Eckart, A., et al. 1992, Nature, 355, 526

Eckart, A., \& Genzel, R. 1997, MNRAS, 284, 576

Eckart, A., Genzel, R., Hofmann, R., Sams, B. J., \& Tacconi-Garman, L. E. 1995, ApJ, 445, L23

Esin, A. A. 1997, ApJ, 482, 400

Esin, A. A., McClintock, J. E., \& Narayan, R. 1997, ApJ, in press (astro-ph/9705237)

Fabian, A. C., \& Rees, M. J., 1995, MNRAS, 277, L55

Falcke, H., 1996, in IAU Symp. 169, Unsolved Problems of the Milky Way, eds. L. Blitz \& P. J. Teuben (Dordrecht: Kluwer), 163

Falcke, H., Biermann, P. L., Duschl, W. J., Mezger, P. G., 1993a, A\&A, 270, 102

Falcke, H., Mannheim, K., \& Biermann, P. L. 1993b, A\&A, 278, L1 
Falcke, H., \& Melia, F., 1997, ApJ, 479, 740

Frank, J., King, A., \& Raine, D. 1992, Accretion Power in Astrophysics, (Cambridge: Cambridge Univ. Press)

Gammie, C., \& Popham, R. 1997, ApJ, in press

Genzel, R., \& Townes, C. H. 1987, ARAA, 25, 377

Genzel, R., Hollenbach, D., \& Townes, C. H. 1994, Rep. Prog. Phys., 57, 417

Gezari, D., Ozernoy, L., Varosi, F., McCreight, D., \& Joyce, R., 1994, in The Nuclei of Normal Galaxies, eds. R. Genzel \& A. I. Harris (Dordrecht: Kluwer), 427

Goldwurm, A. et al. 1994, Nature, 371, 589

Hameury, J. M., Lasota, J. P., McClintock, J. E., \& Narayan, R., 1997, ApJ, in press (astro-ph/9703095)

Hawley, J. F., Gammie, C. F., \& Balbus, S. A., 1996, ApJ, 464, 690

Haller, J. W., Rieke, M. J., Rieke, G. H., Tamblyn, P., Close, L., \& Melia, F., 1996, 456, 194 (Erratum, 1996, ApJ, 468, 955)

Ichimaru, S., 1977, ApJ, 214, 840

Jaroszynski, M., \& Kurpiewski, A. 1997 A\&A, in press (astro-ph 9705044).

Lasota, J. P., Abramowicz, M. A., Chen, X., Krolik, J., Narayan, R., \& Yi, I., 1996, ApJ, 462,142

Kato, S., Abramowicz, M. A., \& Chen, X. 1996, PASJ, 48, 67

Krichbaum, T. P., et al., 1993, A\&A, 274, L37

Koyama, K. et al. 1996, PASJ, 48, 249

Maeda, Y. et al., 1996, PASJ, 48, 417

Mahadevan, R. 1997, ApJ, 477, 585

Mahadevan, R, Narayan, R., \& Krolik, J. 1997, ApJ, 486, in press (astro-ph/9704018)

Mahadevan, R., Narayan, R., \& Yi, I. 1997, ApJ, 465, 327

Mahadevan, R., \& Quataert, 1997, ApJ, 490, in press astro-ph/9705067)

Manmoto, T., Mineshige, S., \& Kusunose, M. 1997, ApJ, in press

Marcaide, J. M. et al., 1992, A\&A, 258, 295

Mastichiadis, A., \& Ozernoy, L. M., 1994, ApJ, 426, 599-603

Melia, F. 1992, ApJL, 387, L25 
Melia, F. 1994, ApJ, 426, 577

Menten, K. M., Reid, M. J., Eckart, A., \& Genzel, R. 1997, ApJL, 475, L111

Merck, M., et al. 1996, A\&A Sup., 120, 465

Mezger, P. G., 1994, in The Nuclei of Normal Galaxies, eds. R. Genzel \& A. I. Harris (Dordrecht: Kluwer), 415

Mezger, P. G, Duschl, W. J., \& Zylka, R., 1996, ARA\&A, 7, 289

Nakamura, K. E., Kusunose, M., Matsumoto, R., \& Kato, S. 1997, PASJ, in press

Narayan, R. 1996, in Physics of Accretion Disks, ed. S. Kato, S. Inagaki, S. Mineshige \& J. Fukue (Gordon \& Breach), 15

Narayan, R. 1997, Proc. IAU Colloq. 163, Accretion Phenomena and Related Outflows, A.S.P. Conf. Series, eds. D. T. Wickramasinghe, L. Ferrario, G. V. Bicknell, in press (astro-ph/9611113)

Narayan, R., Barret, D., \& McClintock, J. E. 1997, ApJ, 482, 448

Narayan, R., Garcia, M. R., \& McClintock, J. E. 1997, ApJ, 478, L79

Narayan, R., Kato, S., \& Honma, F. 1997, ApJ, 476, 49

Narayan, R., McClintock, J. E., \& Yi, I. 1996, ApJ, 457, 821

Narayan, R., \& Yi, I. 1994, ApJ, 428, L13

Narayan, R., \& Yi, I. 1995a, ApJ, 444, 231

Narayan, R., \& Yi, I. 1995b, ApJ, 452, 710

Narayan, R., Yi, I., \& Mahadevan, R. 1995, Nature, 374, 623

Narayan, R., Yi, I., \& Mahadevan, R. 1996, A\&A Sup., 120, 287

Pavlinsky, M. N., Grebenev, S. A., \& Sunyaev, R. A., 1992, Soviet Astr. Lett., 18, 116

Pavlinsky, M. N., Grebenev, S. A., \& Sunyaev, R. A., 1994, ApJ, 425, 110

Peitz, J., \& Appl, S., 1997, MNRAS, 286, 681

Phinney, E. S., 1981, in Plasma Astrophysics, ed. T. D. Guyenne \& G. Levy (ESA SP-161), 337

Popham, R., \& Gammie, C. 1997, ApJ, submitted

Poutanen, J., \& Svensson, R. 1996, ApJ, 470, 249

Predehl, P., \& Trumper, J. 1994, A\&A, 290, L29

Rogers, A. E. E., et al., 1994, ApJ, 434, L59 
Rosa, M. R., Zinnecker, H., Moneti, A., \& Melnick, J., A\&A, 257, 515

Rees, M. J. 1982, in The Galactic Center, ed. G. R. Riegler \& R. D. Blandford, AIP: New York, p166

Rees, M. J., Begelman, M. C., Blandford, R. D., \& Phinney, E. S. 1982, Nature, 295, 17

Reynolds, C. S., Di Matteo, T., Fabian, A. C., Hwang, U., \& Canizares, C. R., 1996, MNRAS, 283, L111

Serabyn, E., Carlstrom, J. E., \& Scoville, N. Z., 1992, ApJ, 401, L87

Serabyn, E., Carlstrom, J., Lay, O., Lis, D. C., Hunter, T. R., \& Lacy, J. H., 1997, ApJ, submitted

Shapiro, S.L., Lightman, A.P., \& Eardley, D.M. 1976, ApJ, 204, 187

Skinner, G. K. et al., 1987, Nature, 330, 544

Stolovy, S. R., Hayword, T. L., \& Herter, T., 1996, ApJ, 470, L45

Telesco, C. M, Davidson, J. A., \& Werner, M. W., 1996, ApJ, 456, 541

Watson, M. G., Willingale, R., Grindlay, J. E., Hertz, P., 1981, ApJ, 250, 142

Wright, M. C. H., Genzel, R., Güsten, R., Jaffe, T., 1987, in The Galactic Center AIP conf. Proc, ed. D. C. Backer, 133

Yusef-Zadeh, F., Morris, M., \& Ekers, R. D., 1990, Nature, 348, 45

Zhao, J., Ekers, R. D., Goss, W. M., Lo, K. Y., \& Narayan, R., 1989, in The Center of the Galaxy, ed. M. Morris, 535

Zhao, J., Goss, W. M., Lo, K. Y., \& Ekers, R. D., 1992, in Relationships Between Active Galactic Nuclei and Starburst Galaxies, ed. A. Filippenko, 31

Zylka, R., \& Mezger, 1988, A\&A, 190, L25

Zylka, R., Mezger, P. G., \& Lesch, H., 1992, A\&A, 261, 119

Zylka, R., Mezger, Ward-Thompson, D., Duschl, W. J., \& Lesch, H., 1995, A\&A, 297, 83

This preprint was prepared with the AAS $\mathrm{IAT}_{\mathrm{E}} \mathrm{X}$ macros v4.0. 


\section{Figure Captions}

Figure 1. The open and filled circles represent various flux measurements and upper limits of Sgr $\mathrm{A}^{*}$. We consider the filled circles to be more important as model constraints. The box at $1 \mathrm{keV}$ represents the uncertainty in the X-ray flux. The solid line is our baseline ADAF model with the following parameters: $M=2.5 \times 10^{6} M_{\odot}, \alpha=0.3, \beta=0.5, \delta=0.001$. The Eddington-scaled mass accretion rate $\dot{m}$ has been adjusted to fit the X-ray flux, giving $\dot{m}=1.3 \times 10^{-4}$. The peak at the left is due to synchrotron radiation, the next peak is due to Compton scattering, the peak between $10-100 \mathrm{keV}$ is due to bremsstrahlung and the peak above $100 \mathrm{MeV}$ is due to pion production. The long-dashed line is a model in which the pion peak has been artificially raised by about an order of magnitude to fit the data. The dotted line is the spectrum corresponding to a standard thin accretion disk

with $\dot{m}=10^{-4}$ while the short-dashed line is a thin disk with $\dot{m}=10^{-9}$. Neither of these models is satisfactory.

Figure 2. (a) Spectra corresponding to five models, showing the effect of various approximations; see the text for details. (b) Electron temperature profiles corresponding to the same five models. The models represented with long-dashed and solid lines have nearly identical temperatures.

Figure 3. (a) The solid line is the baseline model shown in Fig. 1, with a black hole mass of $M=2.5 \times 10^{6} M_{\odot}$. The dotted line corresponds to a model with $M=8.3 \times 10^{5} M_{\odot}$ and the dashed line corresponds to $M=7.5 \times 10^{6} M_{\odot}$. (b) The solid line is the baseline model with a mass accretion rate $\dot{m}=1.3 \times 10^{-4}$. The dotted, short-dashed, long-dashed and dash-dotted lines corresponds to models with $\dot{m}=6.4 \times 10^{-5}, 9.0 \times 10^{-5}, 1.8 \times 10^{-4}, \dot{m}=2.5 \times 10^{-4}$, respectively.

Figure 4. (a) The solid line is the baseline model shown in Fig. 1, with $\alpha=0.3$. The dotted, short-dashed and long-dashed lines correspond to models with $\alpha=0.1,0.2,0.4$ respectively. (b) The solid line is the baseline model shown in Fig. 1, with $\beta=0.5$. The dotted, short-dashed, long-dashed lines and dot-dashed lines correspond to models with $\beta=0.3,0.4,0.6,0.7$ respectively. (c) Electron temperature profiles corresponding to the models shown in (b). (d) The solid line is the baseline model shown in Fig. 1, with $\delta=$ 0.001. The dotted, short-dashed, long-dashed and dot-dashed lines correspond to models with $\delta=0,0.003162,0.01,0.03162$ respectively.

Figure 5. (a) The solid line is the baseline model shown in Fig. 1. The dotted line corresponds to a model in which the temperature of the electrons has been fixed at $T_{e}=2 \times 10^{9}$ $\mathrm{K}$ over the radius range $r=20-1000$. This model fits the low frequency radio data better. (b) Electron temperature profiles corresponding to the two models shown in (a). 
Table 1: Sgr A*: Radio and NIR Observations (Distance $=8.5 \mathrm{kpc}$ )

\begin{tabular}{|c|c|c|c|c|c|}
\hline $\begin{array}{l}\nu \\
\mathrm{Hz}\end{array}$ & $\begin{array}{c}\lambda \\
\mu \mathrm{m}\end{array}$ & $\begin{array}{l}\theta \\
\prime \prime\end{array}$ & $\begin{array}{l}S_{\nu} \\
\text { Jy }\end{array}$ & 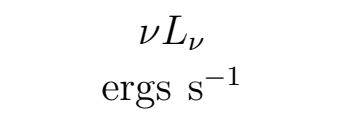 & Ref. \\
\hline 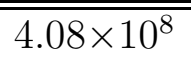 & $\overline{c 735294}$ & 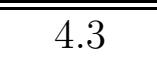 & $\bar{s} \leq 0.05$ & $\leq 1.76 \times 10^{30}$ & $\begin{array}{l}\text { Davies et al. } 76 \\
\end{array}$ \\
\hline $9.6 \times 10^{8}$ & 312500 & 10 & 0.29 & $2.41 \times 10^{31}$ & Davies et al. 76 \\
\hline $9.6 \times 10^{8}$ & 312500 & 10 & 0.27 & $2.24 \times 10^{31}$ & Davies et al. 76 \\
\hline $9.6 \times 10^{8}$ & 312500 & 10 & 0.30 & $2.49 \times 10^{31}$ & Davies et al. 76 \\
\hline $1.66 \times 10^{9}$ & 180722 & 2.5 & 0.56 & $8.04 \times 10^{31}$ & Davies et al. 76 \\
\hline $1.5 \times 10^{9}$ & 200000 & $\sim 1.2$ & 0.8 & $1.04 \times 10^{32}$ & Backer 82 \\
\hline $1.5 \times 10^{9}$ & 200000 & $\sim 1.2$ & 0.3 & $3.89 \times 10^{31}$ & Zhao et al. 89 \\
\hline $2.3 \times 10^{9}$ & 130000 & 0.2 & $1.1 \pm 0.1$ & $(2.2 \pm 0.2) \times 10^{32}$ & Marcaide et al. 92 \\
\hline $2.7 \times 10^{9}$ & 110000 & $\sim 0.65$ & .73 & $1.7 \times 10^{32}$ & Backer 82 \\
\hline $2.7 \times 10^{9}$ & 110000 & $\sim 0.65$ & .42 & $9.78 \times 10^{31}$ & Brown \& Lo 82 \\
\hline $5.0 \times 10^{9}$ & 60000 & $\sim 0.35$ & 1.13 & $1.45 \times 10^{32}$ & Zhao et al. 89 \\
\hline $5.0 \times 10^{9}$ & 60000 & $\sim 0.35$ & 0.55 & $7.13 \times 10^{31}$ & Zhao et al. 89 \\
\hline $8.1 \times 10^{9}$ & 37000 & $\sim 0.22$ & 0.9 & $6.23 \times 10^{32}$ & Backer 82 \\
\hline $8.1 \times 10^{9}$ & 37000 & $\sim 0.22$ & 0.58 & $4.01 \times 10^{32}$ & Brown \& Lo 82 \\
\hline $8.3 \times 10^{9}$ & 36000 & 0.016 & $1.2 \pm 0.1$ & $(8.6 \pm 0.7) \times 10^{32}$ & Marcaide et al. 92 \\
\hline $8.4 \times 10^{9}$ & 35714 & $\sim 0.21$ & 1.07 & $7.77 \times 10^{32}$ & Zhao et al. 92 \\
\hline $8.4 \times 10^{9}$ & 35714 & $\sim 0.21$ & 0.55 & $3.99 \times 10^{32}$ & Zhao et al. 92 \\
\hline $1.5 \times 10^{10}$ & 20000 & $\sim 0.12$ & 1.64 & $2.1 \times 10^{33}$ & Zhao et al. 92 \\
\hline $1.5 \times 10^{10}$ & 20000 & $\sim 0.12$ & .68 & $8.7 \times 10^{32}$ & Zhao et al. 92 \\
\hline
\end{tabular}


Table 1: Sgr A*: Radio and NIR Observations (continued).

\begin{tabular}{|c|c|c|c|c|c|}
\hline $\begin{array}{l}\nu \\
\mathrm{Hz}\end{array}$ & $\begin{array}{c}\lambda \\
\mu \mathrm{m}\end{array}$ & $\begin{array}{l}\theta \\
\prime \prime\end{array}$ & $\begin{array}{l}S_{\nu} \\
\mathrm{Jy}\end{array}$ & $\begin{array}{c}\nu L_{\nu} \\
\operatorname{ergs} \mathrm{s}^{-1}\end{array}$ & Ref. \\
\hline $1.5 \times 10^{10}$ & 20000 & $0.12 \times 0.24$ & $\overline{~ 1.15+0.01}$ & 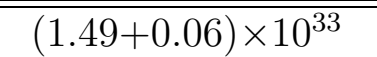 & "Yusef-Zadeh et al. 90 \\
\hline $2.2 \times 10^{10}$ & 13600 & $\sim 0.08$ & 2.1 & $3.99 \times 10^{33}$ & Zhao et al. 92 \\
\hline $2.2 \times 10^{10}$ & 13600 & $\sim 0.08$ & 0.8 & $1.52 \times 10^{33}$ & Zhao et al. 92 \\
\hline $2.2 \times 10^{10}$ & 13500 & $1.8 \times 10^{-3}$ & $1.07 \pm 0.15$ & $(2.04 \pm 0.29) \times 10^{33}$ & Alberdi et al. 93 \\
\hline $2.3 \times 10^{10}$ & 13000 & $1.8 \times 10^{-3}$ & $1.2 \pm 0.4$ & $(2.4 \pm 0.8) \times 10^{33}$ & Marcaide et al. 92 \\
\hline$\star 4.3 \times 10^{10}$ & 7000 & $0.75 \times 10^{-3}$ & $1.4 \pm 0.1$ & $(5.2 \pm 0.4) \times 10^{33}$ & Krichbaum et al. 94 \\
\hline $8.6 \times 10^{10}$ & 3488 & 0.02 & 1.3 & $9.67 \times 10^{33}$ & Backer 82 \\
\hline $8.6 \times 10^{10}$ & 3488 & $4 \times 8$ & 1.05 & $7.8 \times 10^{33}$ & Wright et al. 87 \\
\hline$\star 8.6 \times 10^{10}$ & 3488 & $0.16 \times 10^{-3}$ & $1.4 \pm 0.2$ & $(1.04 \pm 0.15) \times 10^{34}$ & Rogers et al. 94 \\
\hline $2.2 \times 10^{11}$ & 1350 & $1.9 \times 4.3$ & $2.4 \pm 0.5$ & $(4.57 \pm .95) \times 10^{34}$ & Serabyn et al. 92 \\
\hline $2.3 \times 10^{11}$ & 1300 & 11 & 2.5 & $4.97 \times 10^{34}$ & Zylka \& Mezger 88 \\
\hline $2.3 \times 10^{11}$ & 1300 & 11 & $2.6 \pm 0.6$ & $(5.2 \pm 1.2) \times 10^{34}$ & Zylka et al. 92 \\
\hline $3.5 \times 10^{11}$ & 870 & 8 & $4.8 \pm 1.2$ & $(1.45 \pm 0.36) \times 10^{35}$ & Zylka et al. 92 \\
\hline $3.75 \times 10^{11}$ & 800 & 13 & $3.5 \pm 0.5$ & $(1.13 \pm 0.16) \times 10^{35}$ & Zylka et al. 95 \\
\hline $5.0 \times 10^{11}$ & 600 & 10 & $4.0 \pm 1.2$ & $(5.19 \pm 0.52) \times 10^{35}$ & Zylka et al. 95 \\
\hline $6.7 \times 10^{11}$ & 450 & 7 & $\leq 1.5$ & $\leq 8.69 \times 10^{34}$ & Dent et al. 93 \\
\hline $6.7 \times 10^{11}$ & 450 & 8 & $3.0 \pm 1.0$ & $(1.74 \pm 0.58) \times 10^{35}$ & Zylka et al. 95 \\
\hline $8.6 \times 10^{11}$ & 350 & 11 & $7 \pm 2$ & $(5.2 \pm 1.5) \times 10^{35}$ & Serabyn et al. 97 \\
\hline $8.6 \times 10^{11}$ & 350 & 11 & $\leq 10$ & $\leq 7.4 \times 10^{35}$ & Mezger 1994 \\
\hline $8.6 \times 10^{11}$ & 350 & 30 & $18.5 \pm 9$ & $(1.38 \pm 0.67) \times 10^{36}$ & Zylka et al. 92 \\
\hline $1.0 \times 10^{13}$ & 30 & 8 & $\leq 120$ & $\leq 1.04 \times 10^{38}$ & Zylka et al. 92 \\
\hline
\end{tabular}


Table 1: Sgr A*: Radio and NIR Observations (continued).

\begin{tabular}{lcccll}
\hline $\begin{array}{l}\nu \\
\mathrm{Hz}\end{array}$ & $\mu \mathrm{m}$ & $\begin{array}{c}\theta \\
\prime \prime\end{array}$ & $\begin{array}{c}S_{\nu} \\
\mathrm{Jy}\end{array}$ & $\begin{array}{c}\nu L_{\nu} \\
\operatorname{ergs~s}^{-1}\end{array}$ & Ref. \\
\hline \hline $1.0 \times 10^{13}$ & 30 & 4 & $\leq 20$ & $\leq 1.7 \times 10^{37}$ & Telesco et al. 96 \\
$\star 1.5 \times 10^{13}$ & 20 & 1.6 & $\leq 1$ & $\leq 1.3 \times 10^{36}$ & Gezari et al. 94 \\
$1.56 \times 10^{13}$ & 19.2 & 4 & $\leq 1.4$ & $\leq 1.9 \times 10^{36}$ & Telesco et al. 96 \\
$\star 1.7 \times 10^{13}$ & 18 & $2.3 \times 1.3$ & $\leq 0.3$ & $\leq 4.4 \times 10^{35}$ & in Zylka et al. 92 \\
$2.3-3.6 \times 10^{13}$ & $13-8$ & $2.3 \times 1.3$ & $\leq 0.1$ & $\leq 2.6 \times 10^{35}$ & in Zylka et al. 92 \\
$\star 3.5 \times 10^{13}$ & 8.7 & 0.5 & $\leq 0.1$ & $\leq 3.0 \times 10^{35}$ & Stolovy et al. 96 \\
$\star 1.4 \times 10^{14}$ & 2.2 & 0.15 & $\leq 9 \times 10^{-3}$ & $\leq 1.1 \times 10^{35}$ & Menten et al. 97
\end{tabular}


Table 2: Sgr A*: X-Ray and $\gamma$-Ray Observations (Distance $=8.5 \mathrm{kpc}$ ).

\begin{tabular}{|c|c|c|c|c|c|}
\hline $\begin{array}{l}\text { Energy } \\
\text { Band (EB) }\end{array}$ & $\begin{array}{l}\text { Telescope/ } \\
\text { Instrument }\end{array}$ & $\theta$ & 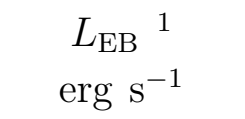 & $\begin{array}{c}\nu L_{\nu} \\
\operatorname{erg~} \mathrm{s}^{-1}\end{array}$ & Ref. \\
\hline $0.8-2.5 \mathrm{keV}$ & ROSAT & $\sim 20^{\prime \prime}$ & $1.55 \times 10^{34}$ & $1.6 \times 10^{34}$ & Predehl \& Trümper $94^{2}$ \\
\hline $2-10 \mathrm{keV}$ & ASCA & $\sim 1^{\prime}$ & $\leq 6.4 \times 10^{35}$ & $\leq 4.8 \times 10^{35}$ & Koyama et al. 96 \\
\hline $35-75 \mathrm{keV}$ & SIGMA & $\sim 15^{\prime}$ & $\leq 3.5 \times 10^{35}$ & $\leq 4.8 \times 10^{35}$ & Goldwrum et al. 94 \\
\hline $75-150 \mathrm{keV}$ & SIGMA & $\sim 15^{\prime}$ & $\leq 2.4 \times 10^{35}$ & $\leq 3.6 \times 10^{35}$ & Goldwrum et al. 94 \\
\hline $30-50 \mathrm{MeV}$ & EGRET & $\sim 1^{\circ}$ & & $\leq 1.8 \times 10^{36}$ & Merck et al. 96 \\
\hline $50-70 \mathrm{MeV}$ & EGRET & $\sim 1^{\circ}$ & & $\leq 1.1 \times 10^{36}$ & Merck et al. 96 \\
\hline $70-100 \mathrm{MeV}$ & EGRET & $\sim 1^{\circ}$ & & $\leq 8.3 \times 10^{35}$ & Merck et al. 96 \\
\hline $100-150 \mathrm{MeV}$ & EGRET & $\sim 1^{\circ}$ & & $\leq 6.2 \times 10^{35}$ & Merck et al. 96 \\
\hline $150-300 \mathrm{MeV}$ & EGRET & $\sim 1^{\circ}$ & & $\left(4.9_{-2.1}^{+2.0}\right) \times 10^{35}$ & Merck et al. 96 \\
\hline $300-500 \mathrm{MeV}$ & EGRET & $\sim 1^{\circ}$ & & $\left(1.2_{-0.26}^{+0.24}\right) \times 10^{36}$ & Merck et al. 96 \\
\hline $500-1000 \mathrm{MeV}$ & EGRET & $\sim 1^{\circ}$ & & $\left(1.4_{-0.28}^{+0.28}\right) \times 10^{36}$ & Merck et al. 96 \\
\hline $1-2 \mathrm{GeV}$ & EGRET & $\sim 1^{\circ}$ & & $\left(1.7_{0.28}^{+0.28}\right) \times 10^{36}$ & Merck et al. 96 \\
\hline $2-4 \mathrm{GeV}$ & EGRET & $\sim 1^{\circ}$ & & $\left(2.2_{-0.62}^{+0.82}\right) \times 10^{36}$ & Merck et al. 96 \\
\hline $4-10 \mathrm{GeV}$ & EGRET & $\sim 1^{\circ}$ & & $\left(8.3_{-4.7}^{+4.2}\right) \times 10^{35}$ & Merck et al. 96 \\
\hline
\end{tabular}




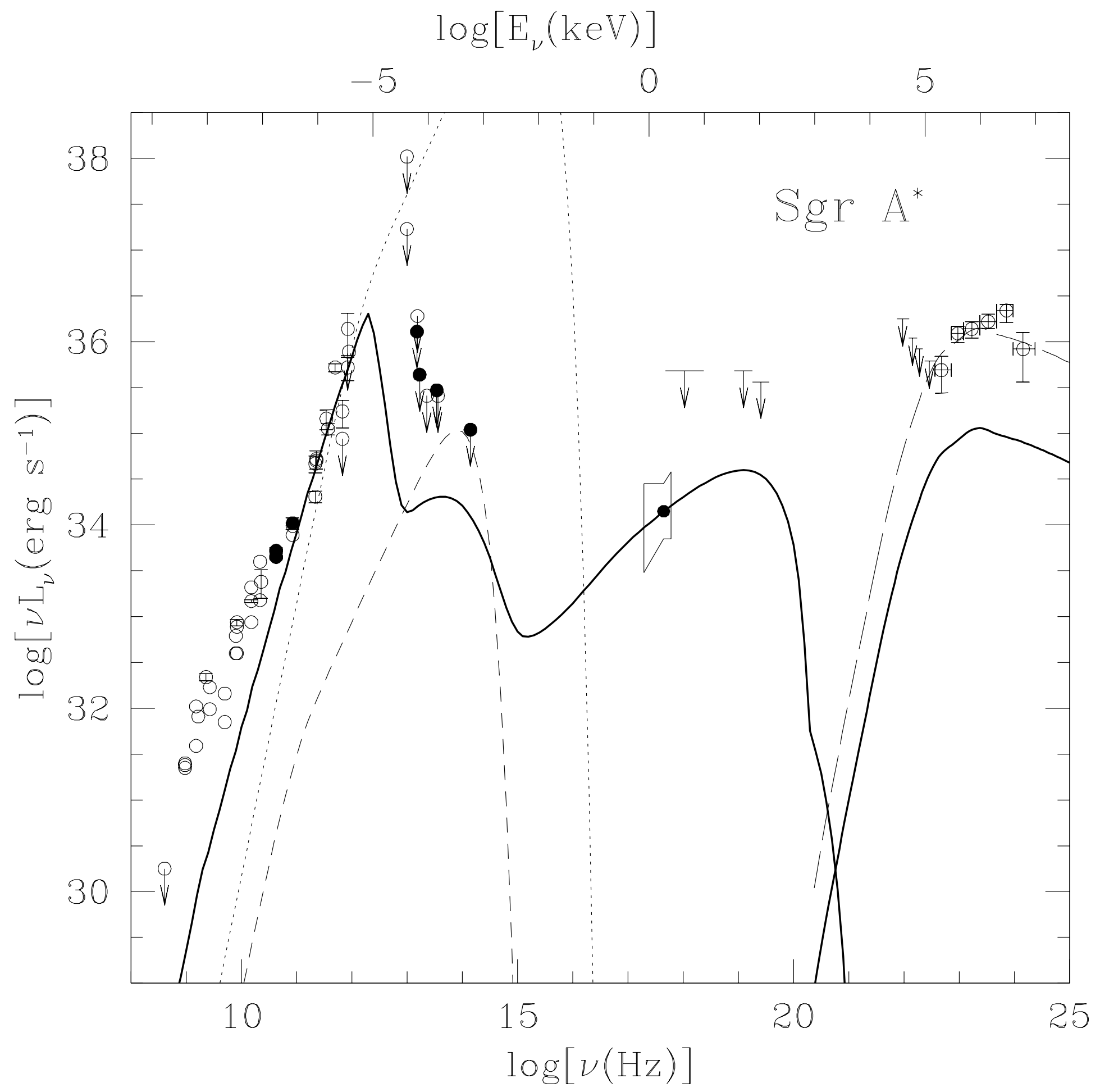

Figure 1 

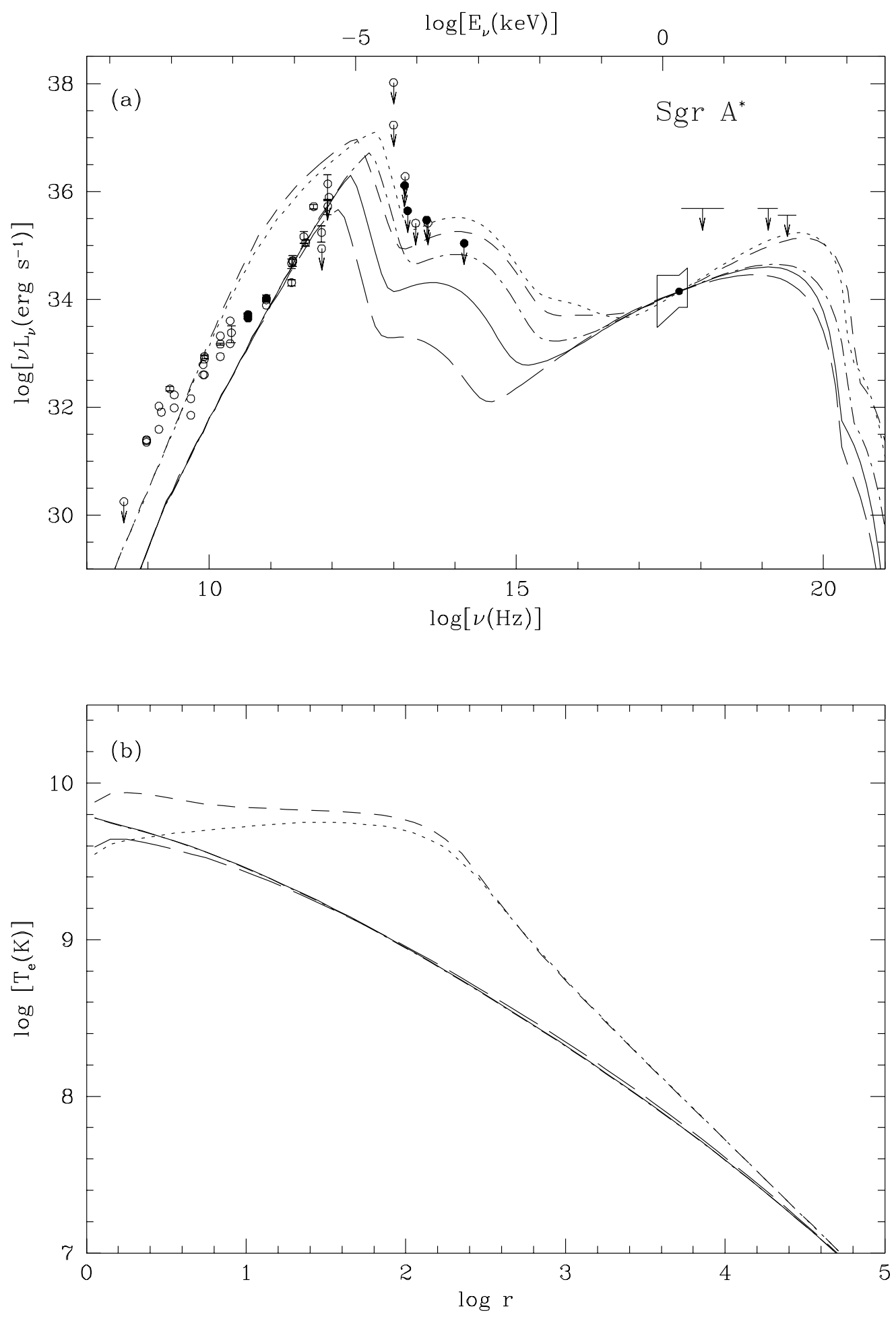

Figure 2 a,b 

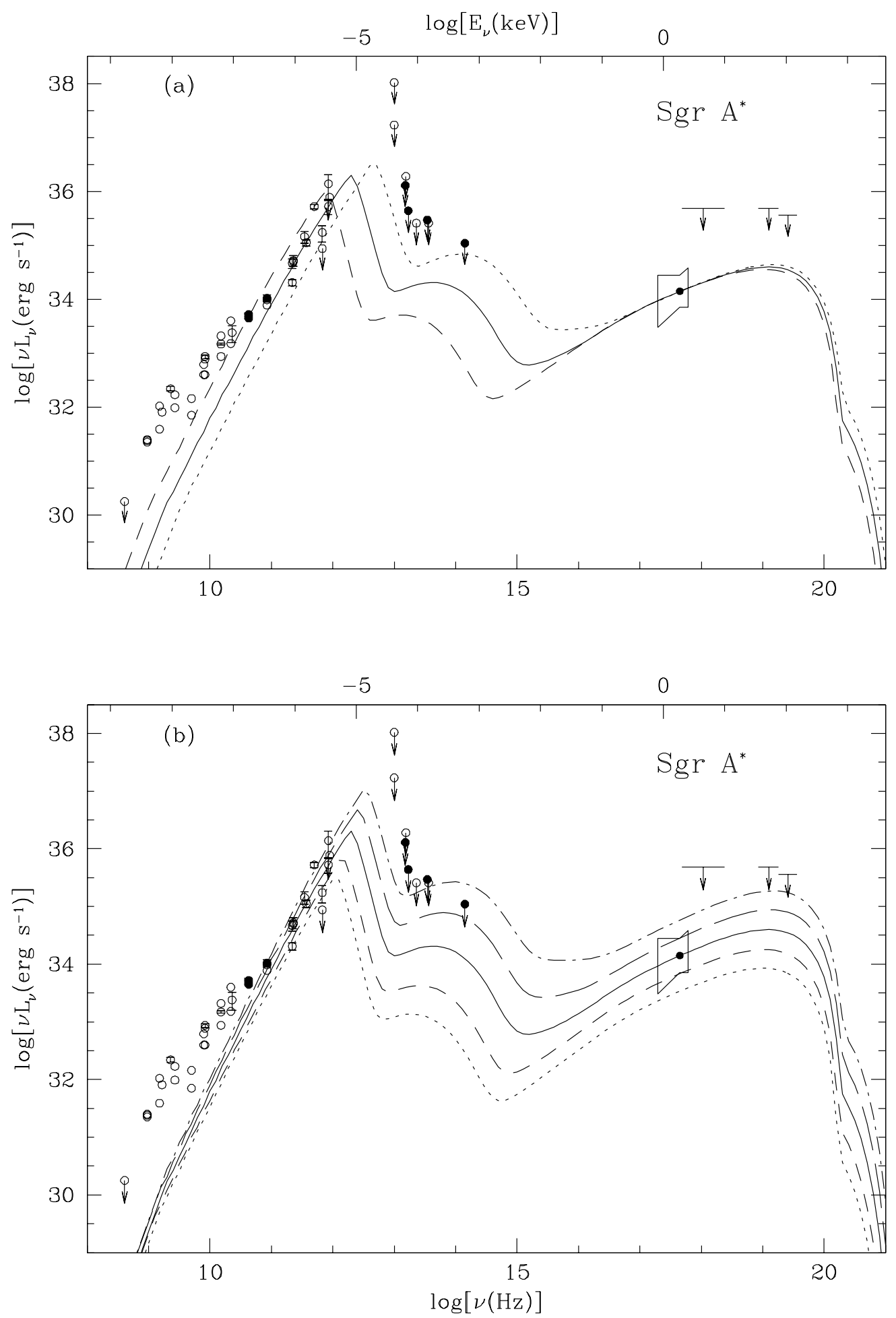

Figure $3 a, b$ 

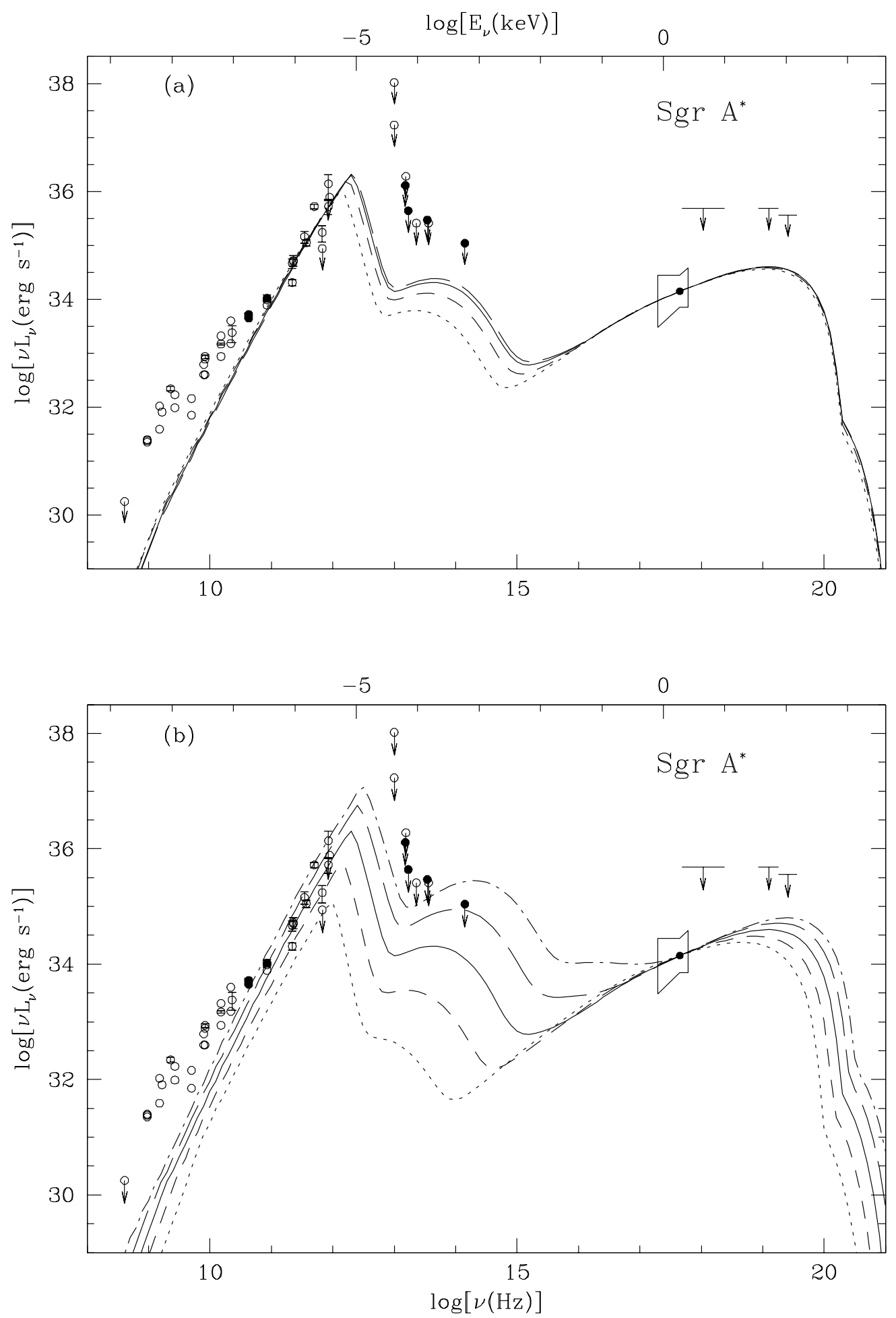

Figure 4 a,b 

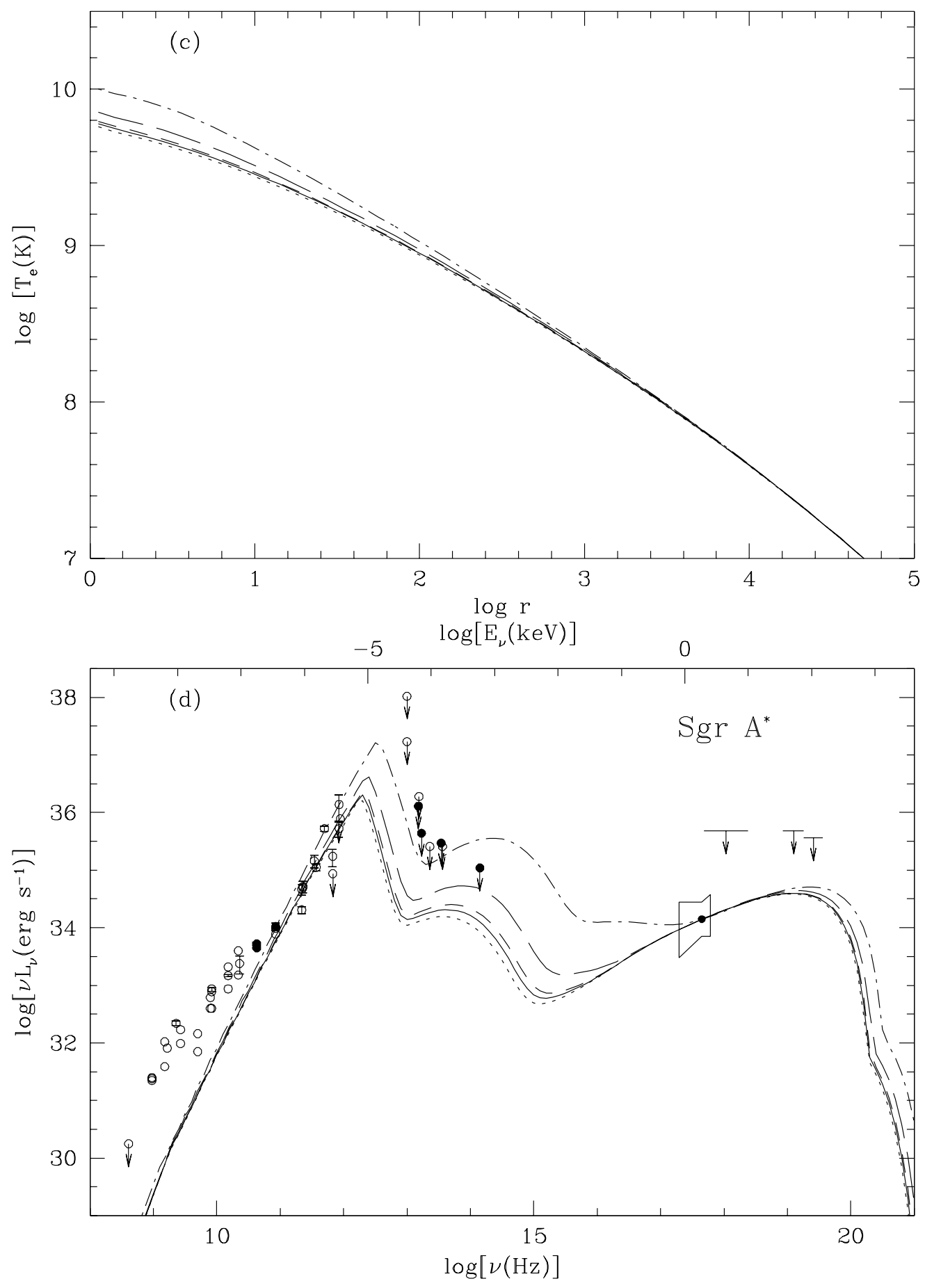

Figure 4 c,d 

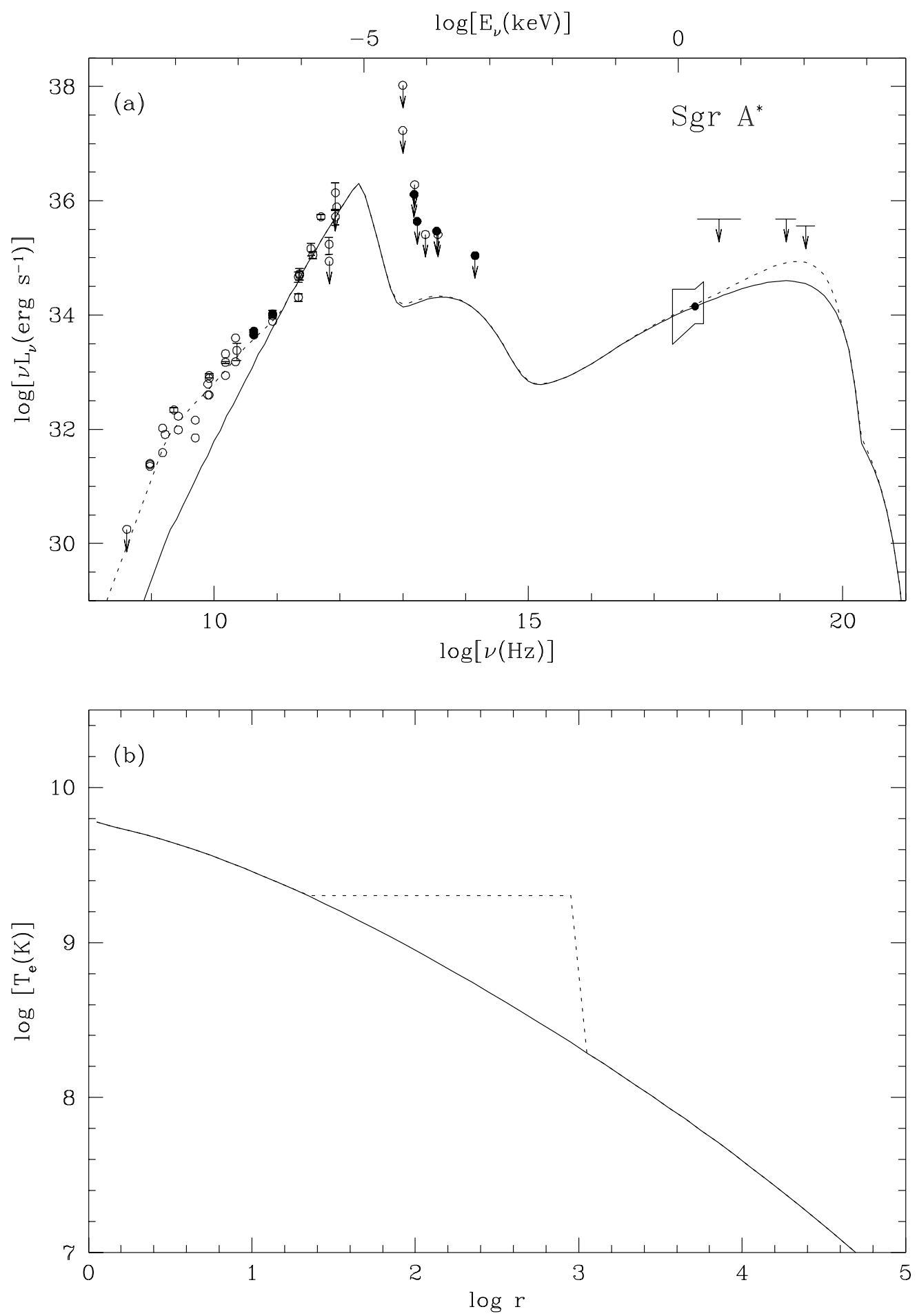

Figure 5 a,b 